\title{
Spectrum Analysis of a Coaxial Dual-Rotor System with Coupling Misalignment
}

\author{
Hongxian Zhang $\mathbb{D}^{1,2}$ Liangpei Huang ${ }^{1},{ }^{1}$ Xuejun Li $\mathbb{D}^{\circ},{ }^{3}$ Lingli Jiang $\left(\mathbb{D},{ }^{3}\right.$ Dalian Yang, \\ Fanyu Zhang, ${ }^{1}$ and Jingjing Miao ${ }^{1}$
}

${ }^{1}$ Hunan Provincial Key Laboratory of Health Maintenance for Mechanical Equipment, Hunan University of Science and Technology, Xiangtan 411201, China

${ }^{2}$ Lushan College of Guangxi University of Science and Technology, Liuzhou 454006, China

${ }^{3}$ School of Mechanical \& Electrical Engineering, Foshan University, Foshan 528000, China

Correspondence should be addressed to Xuejun Li; hnkjdxlxj@163.com

Received 6 December 2019; Revised 7 May 2020; Accepted 13 June 2020; Published 10 July 2020

Academic Editor: Jussi Sopanen

Copyright (c) 2020 Hongxian Zhang et al. This is an open access article distributed under the Creative Commons Attribution License, which permits unrestricted use, distribution, and reproduction in any medium, provided the original work is properly cited.

\begin{abstract}
The finite element model of a dual-rotor system was established by Timoshenko beam element. The dual-rotor system is a coaxial rotor whose supporting structure is similar to that of an aero-engine rotor system. The inner rotor is supported by three bearings, which makes it a redundantly supported rotor. The outer rotor connects the inner rotor by an intershaft bearing. The spectrum characteristics of the dual-rotor system under unbalanced excitation and misalignment excitation were analysed in order to study the influence of coupling misalignment of the inner rotor on the spectral characteristics of the rotor system. The results indicate that the vibration caused by the misaligned coupling of the inner rotor will be transmitted to the outer rotor through the intershaft bearing. Multiple harmonic frequency components, mainly $1 x$ and $2 x$, will be excited by the coupling misalignment. The amplitudes of all harmonic frequencies increase with the misalignment in both the inner and outer rotors. The vibration level of the outer rotor affected by the misalignment is lower than that of the inner rotor because it is far from the misaligned coupling. Harmonic resonance occurs when any harmonic frequencies of the misalignment response coincide with a natural frequency of the system. In order to verify the theoretical model, experiments are performed on a test rig. Both the experimental and simulation results are in good accordance with each other.
\end{abstract}

\section{Introduction}

Rotor misalignment is one of the most common difficulties in the operation of rotating machinery. A misalignment rotor negatively influences the rolling, sealing, and coupling parts and can also produce eccentricity in the air gap. Therefore, a misaligned machine is more prone to failure due to the increased loads on bearings and couplings. In general, the misalignment in a rotor system is unavoidable and cannot be completely eliminated. Furthermore, it can disguise itself very well on industrial rotating machinery, which makes it not easy to be detected. Even if the alignment looks good when you do an offline check, a running misalignment may occur. What we witness is the secondary effects of misalignment as it slowly damages the machinery over long periods of time. Hence, the ability to clearly diagnose the presence of misalignment using vibration analysis can be vital in reducing costly machine unscheduled downtime. Vibration based identification of faults, such as rolling bearing fault [1], gear fault [2], crack [3], and rub [4], is welldeveloped and widely used in practice. However, because more attention is paid to the study of the misalignment of the series connection rotor (such as steam turbine rotor) than that of a coaxial rotor, the misalignment of a coaxial dual-rotor system remains as an outstanding area, where the basic understanding is somewhat lacking.

In the fault diagnosis of misalignment, Lees et al. [5] proposed a method that can reliably estimate both the rotor 
unbalance and misalignment from measured vibration during a single machine run-down. Lal and Tiwari [6] developed a model based approach which can estimate the bearing and coupling dynamic parameters of a laboratory test rig as a measure of misalignment. Rybczynski [7] indicated the potential of using trajectory patterns for diagnosing misalignment defects in rotating machines and suggested including sets of trajectory patterns to the knowledge base of a machine diagnostic system. Verucchi et al. [8] studied the techniques that can detect misalignment from the analysis of the motor electrical variable, and the results show that the conclusions drawn for a particular coupling are not necessarily applicable to others. Reddy and Sekhar [9] studied a technique that uses torque measurements to detect and monitor coupling misalignment. Qi et al. [10] proposed a novel method to analyse in time frequency domain under different misalignment conditions, and the experimental results show that the proposed method is able to detect misalignment fault characteristic frequency effectively. Patel et al. [11-13] investigated the influence of misalignment and its type on the forcing characteristics of flexible coupling by the full spectrum.

In terms of the dynamic characteristics of rotor misalignment, Hili et al. [14] and Fakhfakh et al. [15] developed a model with the shaft angular misalignment to identify misalignment from the vibration response. Li et al. [16] and Li et al. [17] discussed the nonlinear dynamic behaviours of a rotor system on misaligned journal bearings. Askarian and Hashemi [18] obtained the effects of unbalance and misalignment on the vibration of a multirotor system. Li et al. [19] presented a theoretical research on the periodic response of an offset disc rotor system with coupling misalignment. Ma et al. [20] discussed the stability of a rotorbearing-coupling system under misalignment and found that the instability rotating speed range increases with misalignment. AL-Hussain [21] indicated that an increase in angular misalignment or mechanical coupling stiffness terms leads to an increase of the model stability region. Yi et al. [22] present a simplified model of misaligned installing matched bearings and found that the amount of misalignment plays an important factor on both the global stability and vibration amplitude for a misaligned matched bearing rotor system. Zhang et al. [23] presented that the support force of a rotor system under misalignment faults appears double vibration and high harmonics frequencies. Nikolajsen [24] studied the relationship between radial bearing misalignment and stability of a multibearing rotor and found that neither perfect alignment nor equal load sharing between bearings can be relied upon to give optimum stability. Gibbons [25] depicted general formulas to calculate the misalignment reaction forces for both gear couplings and flexure couplings in 1976. Sekhar and Prabhu [26] derived the reaction force formulas for the angular misalignment of flexure couplings based on Gibbons formulas. Lee and Lee [27] developed a dynamic model for a misaligned rotor-ball bearing system by the Gibbons-Sekhar formula. Both the experimental and simulation results showed that, as the angular misalignment increases, the whirling orbits tend to collapse toward a straight line and the natural frequency of the misaligned rotor system associated with the misalignment direction increases largely. The reaction forces calculated from the Gibbons-Sekhar formula are the static load. For a rotating shaft, the static load can be decomposed as series dynamic misalignment forces. Prabhakar et al. [28], Jalan et al. [29], Wan et al. [30], and Ma [31] et al. built several rotor bearing system models using the dynamic misalignment forces. All these studies showed that $2 \mathrm{x}$ frequency components occur no matter for angular misalignment or parallel misalignment. Dewelland Mitchell [32] also gave a discussion of the harmonics arising inflexible couplings. Lees [33] illustrated the source of $2 x$ harmonic excitation in misaligned rotors with a rigid coupling uses a purely linear model. Xuand Marangoni $[34,35]$ developed a model of a motor-coupling-rotor system. The torque due to the misalignment of a universal joint was derived in their pair of papers, and their theoretical and experimental studies indicate that the forcing frequencies caused by shaft misalignment are even multiple frequencies of the motor rotation speed.

The research object of the previous studies is the multispan rotor system. However, little information has been done on the misalignment of the coaxial rotor. A coaxial rotor of an aero-engine is different from a series connection rotor of steam turbine in twofold. For one thing, the coaxial shafts corotate or count-rotate at different speeds, for another, the inner rotor which is supported by more than two bearings is a redundant structure. These special structures deserve to be paid more attention. Lu et al. [36] presented a theoretical research on the nonlinear response characteristics of an aero-engine dual-rotor-bearing system with flexible coupling misalignment faults in the inner rotor. $\mathrm{Li}$ et al. [37] described the mechanism and influencing factors of nonlinear properties of a misaligned inner rotor in an aero-engine through Lagrange equations. Wang et al. [38] and Wang et al. [39] et al. developed a multibearing rotor model with misalignment to investigate the dynamic response when the misalignment parameters are uncertain. $\mathrm{Li}$ et al. [40] also investigated the quantification of uncertainty effects on the dynamic responses and vibration characteristics of a multirotor bearing system with the fault of angular misalignment. Ren et al. [41] presented a dynamic model of a flexible rotor system with multisupport in order to solve the bearing misalignment problem of the rotor system in an aero-engine. Previous studies have given detailed discussions to understand the effects of misalignment on a dualrotor system of an aero-engine. However, most of them either took the inner rotor system instead of the whole dual rotors system or showed no experimental evidence to support the theoretical research. Thus, more researches are still required to illustrate the effects of misalignment on a coaxial dual-rotor system.

Spectrum analysis is one of the basic techniques used to conduct misalignment diagnosis. Therefore, the spectrum analysis of an aero-engine multibearing dual-rotor system with coupling misalignment and disk unbalance has been studied by the finite element method (FEM) in the present study. A test rig has a similar bearing supporting structure like a real aero-engine was built to verify the theoretical 
studies. Experiments were performed on the test rig under different intentional misalignment conditions. The study could be useful in the detection of misalignment in the coaxial dual-rotor system diagnosis and in the design of an aero-engine rotor system.

\section{Modelling of a Dual-Rotor-Bearing System with Coupling Misalignment}

A structural diagram of a typical twin-spool jet engine is shown in Figure 1 [42]. LP Comp, HP Comp, HP Turb, and LP Turb denote low pressure compressor, high pressure compressor, high pressure turbine, and low pressure turbine, respectively. The jet engine is a coaxial dual-rotor system. The shaft of the inner rotor is solid and the shaft of the outer rotor is hollow. The two rotors are connected by an intershaft bearing. The intershaft bearing inner race is mounted on the inner rotor while the outer race is mounted on the outer rotor. The outer and inner rotors rotate independently, which can rotate at different rotating speeds and different directions. The rotor constitutive elements are shafts (circular section) and disks. And the inner rotor is supported by three bearings, which makes it a redundantly supported rotor. A coupling is designed to accommodate the misalignment of the inner rotor. The coupling is located at the end of the first span of the inner rotor near the second bearing, which is shown in Figure 2.

2.1. Finite Element Model of the Dual-Rotor System. The dualrotor system shown in Figure 2 is modelled by the FEM [43]. To develop the equations of motion for the rotor bearing system, a stationary coordinate system is defined, which is shown in Figure 3. The stationary coordinate system is consisting of three mutually perpendicular axes-ox, $o y$, and $o z$-intersecting at the point $o$ and fixed in space. The axis of the dual-shaft rotor, in equilibrium, is coincident with the axis $o z$. In the equilibrium position, the rotor lies on a straight line passing through the bearing canters. The axes $o x, o y$, and $o z$, in that order, form a right-handed set, which is defined as follows.

A rotation of a right-handed screw from $o x$ to $o y$ advances it along $o z$ which is assumed to be horizontal. A rotation of a right-handed screw from $o y$ to $o z$ advances it along $o x$, which is perpendicular to the $o y z$-plane. A rotation of a right-handed screw from $o z$ to $o x$ advances it along $o y$ which is assumed to be vertical.

Using the FEM, the rotor is divided into a number of shaft elements with nodes at both ends of each element. The finite shaft element of the dual-rotor system is accomplished by means of the Timoshenko beam. Each shaft element has two nodes, and the node location is shown in Table 1. Disks and bearings are assumed to be attached to the shaft at these nodes where required. The inner rotor is modelled with 15 elements and 16 nodes. The outer rotor is modelled with 8 elements and 9 nodes. Thus, the model has 23 elements and 25 nodes in total. The nodes of the inner rotor are denoted by blue dots and those of the outer rotor are denoted by red squares, which are shown in Figure 2. Only lateral vibrations are considered, so each node has four generalized coordinates (four degrees of freedom per node): transverse displacements in the $x$-and $y$-directions and rotations about the $\mathrm{x}$ - and $y$-axes. The translations of the shaft from the equilibrium position are $u$ and $v$ in the $x$ - and $y$-directions, respectively. The rotations are defined such that $\theta$ is a positive rotation about the $x$-axis and $\varphi$ about the $y$-axis.

The rotors and disks are made from steel. The rotor structure is simplified, and the change of some shaft sections is ignored. The diameters of the shafts are shown in Table 2.

All the five disks are identical. Disk 1 and disk 2 represent a low pressure compressor. Disk 3, disk 4, and disk 5 represent a low pressure turbine, a high pressure compressor, and a high pressure turbine, respectively. Disk 1 , disk 2 , disk 3 , disk 4 , and disk 5 are located at nodes $3,5,13,20$, and 22, respectively. The disk properties are listed in Table 3 .

There are five bearings in the system, namely, bearing 1 , bearing 2 , bearing 3 , bearing 4 , and bearing 5 . Bearing 5 is an intershaft bearing. All bearings are assumed as to be linear and isotropic. Bearing 1, bearing 2, bearing 3 , and bearing 4 are located at nodes $1,7,15$, and 18 , respectively. The intershaft bearing, bearing 5 , is located at node 12 and node 24. The bearings properties are listed in Table 4.

According to the Lagrange equation, the dynamic equations of disk element and shaft element can be derived as follows:

$$
\left\{\begin{array}{l}
\mathbf{M}_{d}^{e} \ddot{\mathbf{q}}_{d}^{e}-\Omega_{d} \mathbf{G}_{d}^{e} \mathbf{q}_{d}^{e}=\mathbf{F}_{d}^{e}, \\
\mathbf{M}_{s}^{e} \ddot{\mathbf{q}}_{s}^{e}-\Omega_{s} \mathbf{G}_{s}^{e} \mathbf{q}_{s}^{e}+\mathbf{K}_{s}^{e} \mathbf{q}_{s}^{e}=\mathbf{F}_{s}^{e},
\end{array}\right.
$$

where $\mathbf{M}_{d}^{e}$ and $\mathbf{M}_{s}^{e}$ are the mass matrixes of the disk element and shaft element, respectively. $\mathbf{q}_{d}^{e}$ and $\mathbf{q}_{s}^{e}$ are the displacement vector of the disk element and shaft element, respectively. Since a disk element has one node, $\mathbf{q}_{d}^{e}$ is a 4dimensional vector. A shaft element has two nodes; $\mathbf{q}_{s}^{e}$ is an 8-dimensional vector. $\Omega_{d}$ and $\Omega_{s}$ are the rotating speeds of the disk element and shaft element, respectively. The rotating speed of the disk element and shaft element on the outer rotor is 1.6 times of that of the disk element and shaft element on the inner rotor. $\mathbf{G}_{d}^{e}$ and $\mathbf{G}_{s}^{e}$ are the gyroscopic matrixes of the disk element and shaft element, respectively. $\mathbf{K}_{s}^{e}$ is the stiffness matrix of a shaft element. $\mathbf{F}_{d}^{e}$ and $\mathbf{F}_{s}^{e}$ are the external forces on the disk element and shaft element, respectively.

The dynamic equations of intershaft bearing and an ordinary bearing are the same, which can be expressed as

$$
-\mathbf{C}_{b}^{e} \dot{\mathbf{q}}_{b}^{e}-\mathbf{K}_{b}^{e} \mathbf{q}_{b}^{e}=\mathbf{F}_{b}^{e},
$$

where $\mathbf{C}_{b}^{e}$ is the damping matrix of bearings. $\mathbf{K}_{b}^{e}$ is the stiffness matrix of bearings. $\mathbf{q}_{b}^{e}$ is the displacement vector of bearings. $\mathbf{F}_{b}^{e}$ is the external force of bearings. The difference between the ordinary bearing and intershaft bearing is that the external force on the intershaft bearing should also include the interaction forces between the two nodes connected by the intershaft bearing. The interaction forces, $F_{\text {intershaft }}$ and $-F_{\text {intershaft }}$, are shown in Figure 4 . The intershaft bearing connects node $j$ of the outer rotor and node $i$ of the inner rotor. The inner and outer rotors interact with each other through the intershaft bearing. The interaction forces 


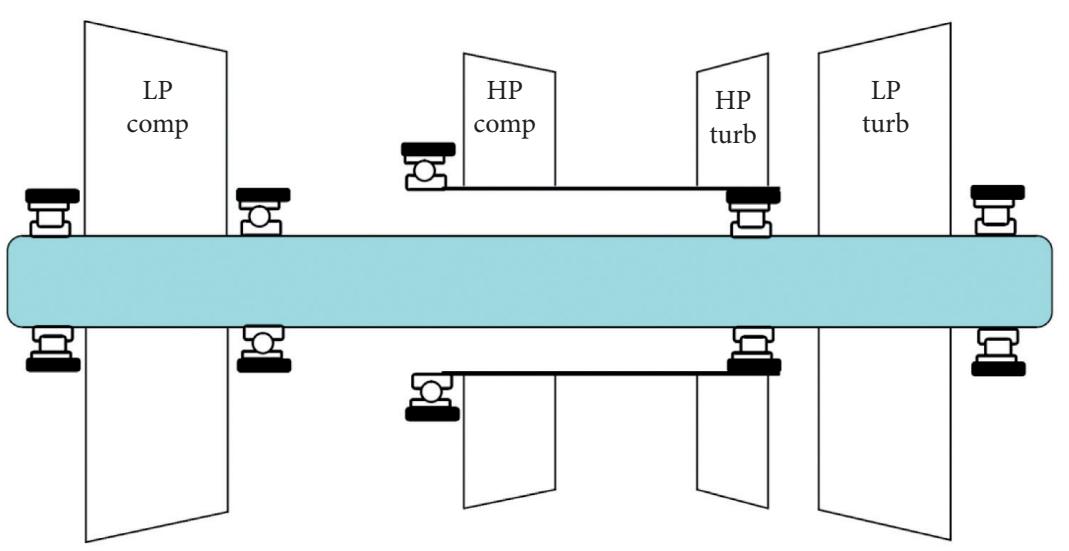

Figure 1: Structural diagram of a typical twin-spool jet engine [42].

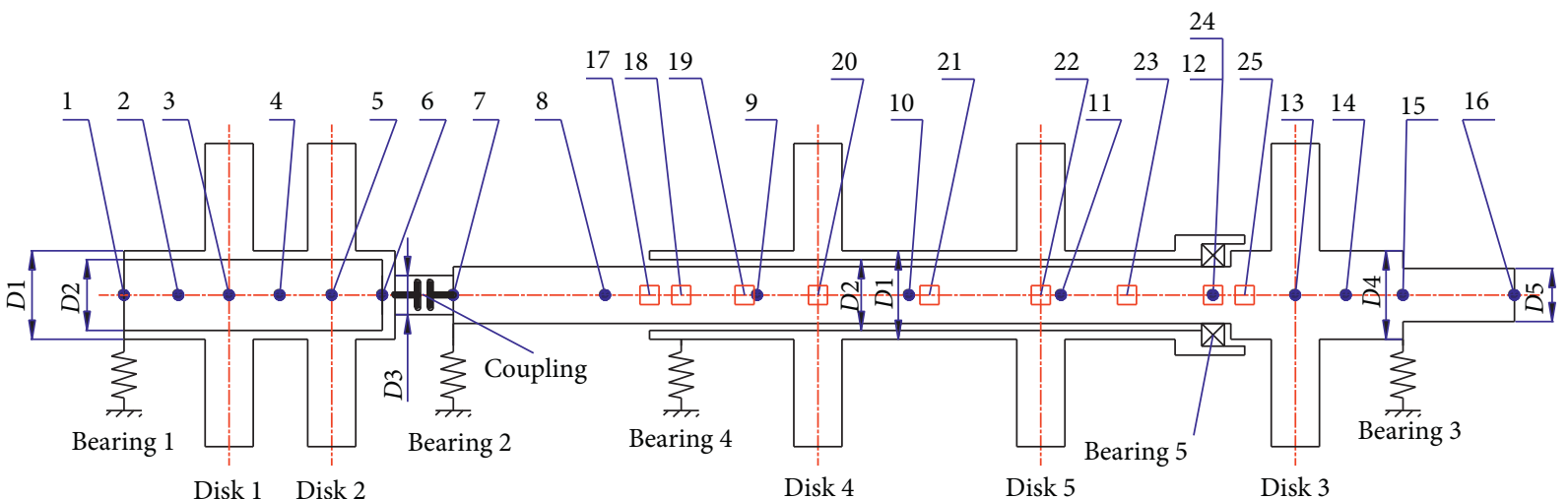

Figure 2: Schematic of the dual-rotor system, nodes, and elements.
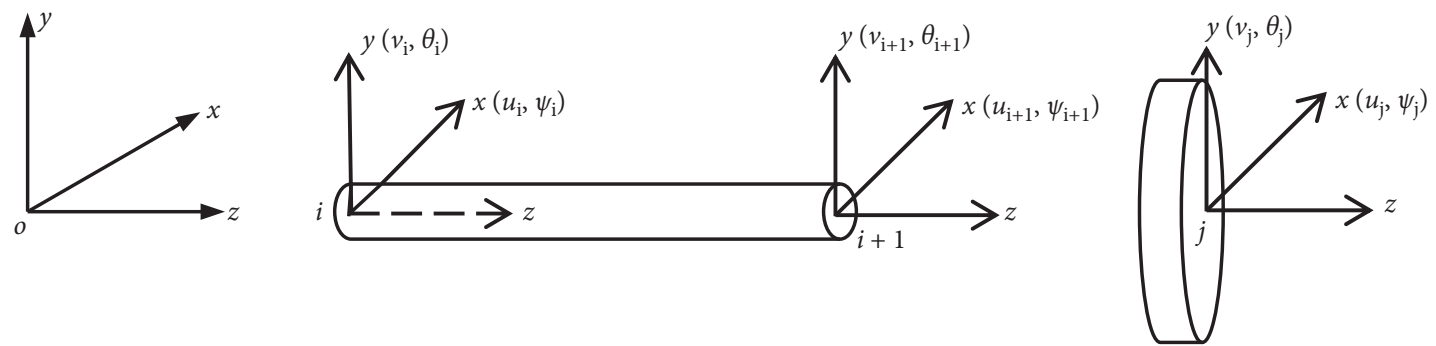

FIGURE 3: Schematic diagram of the coordinate system of the shaft unit.

are the action force and reaction force. Although these two forces are equal in magnitude and opposite in direction, they cannot be cancelled out. These two forces should be calculated as the external forces of the corresponding node, because node $i$ and node $j$ where the intershaft bearing is located are not adjacent.

Considering all the damping, stiffness, and mass matrices of shaft, disk, and bearing, the general dynamic equations of the dual-rotor system can be written as

$$
\mathbf{M} \ddot{\mathbf{q}}+\left(\mathbf{C}+\Omega_{1} \mathbf{G}_{1}-\Omega_{2} \mathbf{G}_{2}\right) \dot{\mathbf{q}}+\mathbf{K q}=\mathbf{F}_{u}+\mathbf{F}_{c}+\mathbf{F}_{g},
$$

where $\mathbf{M}, \mathbf{C}$, and $\mathbf{K}$ are the mass, damping, and stiffness matrixes of the dual-rotor system, respectively. $\mathbf{q}$ is the displacement vector. $\Omega_{1}$ and $\Omega_{2}$ are the rotation speed of the inner rotor and outer rotor, respectively. $\mathbf{G}_{1}$ and $\mathbf{G}_{2}$ are the gyroscopic matrixes of the inner and outer rotor, respectively. $\mathbf{F}_{g}$ is the gravity vector. $\mathbf{F}_{c}$ is the reacting force and moment vector caused by the misaligned coupling, which 
TABle 1: Node location.

\begin{tabular}{|c|c|c|}
\hline Node & Position (m) & Remark \\
\hline 1 & 0.000 & Bearing 1 \\
\hline 2 & 0.043 & \\
\hline 3 & 0.083 & Disk 1 \\
\hline 4 & 0.123 & \\
\hline 5 & 0.164 & Disk 2 \\
\hline 6 & 0.204 & \\
\hline 7 & 0.260 & Bearing 2 \\
\hline 8 & 0.380 & \\
\hline 9 & 0.500 & \\
\hline 10 & 0.620 & \\
\hline 11 & 0.740 & \\
\hline 12 & 0.860 & Bearing 5 \\
\hline 13 & 0.925 & Disk 3 \\
\hline 14 & 0.965 & \\
\hline 15 & 1.010 & Bearing 3 \\
\hline 16 & 1.098 & \\
\hline 17 & 0.415 & \\
\hline 18 & 0.440 & Bearing 4 \\
\hline 19 & 0.490 & \\
\hline 20 & 0.548 & Disk 4 \\
\hline 21 & 0.636 & \\
\hline 22 & 0.724 & Disk 5 \\
\hline 23 & 0.792 & \\
\hline 24 & 0.860 & Bearing 5 \\
\hline 25 & 0.885 & \\
\hline
\end{tabular}

TABLE 2: Diameters of the shafts.

\begin{tabular}{lccccc}
\hline Parameters & $D 1$ & $D 2$ & $D 3$ & $D 4$ & $D 5$ \\
\hline Values $(\mathrm{m})$ & 0.07 & 0.056 & 0.035 & 0.07 & 0.042 \\
\hline
\end{tabular}

TABle 3: Disk properties for the rotor system.

\begin{tabular}{lcccc}
\hline Parameters & Diameter $(\mathrm{m})$ & Thickness $(\mathrm{m})$ & Elastic modulus $(\mathrm{MPa})$ & Density $(\mathrm{kg} / \mathrm{m} 3)$ \\
\hline Values & 0.24 & 0.038 & $2.06 \mathrm{e} 5$ & 7850 \\
\hline
\end{tabular}

TABLE 4: Bearing properties for the rotor system.

\begin{tabular}{lccc}
\hline Bearing & $K_{x x}(\mathrm{MN} / \mathrm{m})$ & $K_{y y}(\mathrm{MN} / \mathrm{m})$ & Node \\
\hline Bearing 1 & 2.21 & 2.21 & 1 \\
Bearing 2 & 14.5 & 14.5 & 7 \\
Bearing 3 & 2.21 & 2.21 & 15 \\
Bearing 4 & 9.29 & 9.29 & 18 \\
Bearing 5 & 25.1 & 25.1 & 12 and 24
\end{tabular}

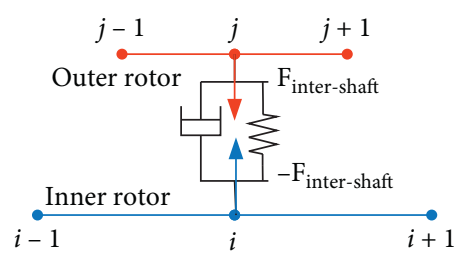

FIGURE 4: Force analysis diagram of the intershaft bearing. will be derived in Section 2.2. $\mathbf{F}_{u}$ is the vectors of the unbalance excitation of the rotor system. $\mathbf{F}_{u}$ can be written as

$$
\begin{aligned}
& \mathbf{F}_{u}=\left[\begin{array}{llll}
\mathbf{F}_{u}^{1} & \mathbf{F}_{u}^{2} & \cdots & \mathbf{F}_{u}^{25}
\end{array}\right]^{\mathrm{T}}, \\
& \mathbf{F}_{u}^{1}=\mathbf{F}_{u}^{2}=\cdots=\mathbf{F}_{u}^{i-1}=\mathbf{F}_{u}^{i+1}=\cdots=\mathbf{F}_{u}^{25}=\left[\begin{array}{llll}
0 & 0 & 0 & 0
\end{array}\right], \\
& \mathbf{F}_{u}^{i}=\left[\begin{array}{llll}
m_{i} \varepsilon_{i}\left(\Omega_{i}\right)^{2} \cos \left(\Omega_{i} t\right) m_{i} \varepsilon_{i}\left(\Omega_{i}\right)^{2} \sin \left(\Omega_{i} t\right) & 0 & 0
\end{array}\right],
\end{aligned}
$$

where $\mathbf{F}_{u}^{i}$ are the out-of-balance forces at node $i . m_{i}$ is the mass of the disk and the shaft attached to node $i$. $\varepsilon_{i}$ is the eccentricity distance from the centre of mass to the shaft centre line at node $i . \Omega_{i}$ is the rotation speed of the unbalance disk.

2.2. Reacting Forces and Moments Caused by Coupling Misalignment. The effects of misalignment on a rotor system depend on how the reaction forces accommodate the 
misalignment. There are many factors that affect the reacting forces and moments of a misaligned coupling, and the structure of the coupling is one of the important factors. [8] Figure 5 shows one kind of coupling used in an aero-engine rotor system [37]. The coupling is used to assemble a low pressure fan rotor and a low pressure turbine rotor in an aeroengine dual-rotor system. The coupling is a spline coupling having some radial locating surfaces and a hexagon nut. The special structure stiffens the spline coupling stronger than a common gear coupling and softer than the same diameter shaft. Moreover, a splined joint instead of a fixed joint makes the spline coupling accommodate misalignment. Therefore, the misalignment of assembly errors and changing of operating temperature occurs indeed in the spline coupling.

According to the structural characteristics of the spline coupling, it can be regarded as a flexure coupling. The reaction forces and moments caused by the spline coupling misalignment can be calculated by the Gibbons-Sekhar formula $[25,26]$, which are shown in Figure 6. The formula can be given as follows.

For parallel misalignment,

$$
\left\{\begin{array}{l}
F_{x 2}=\left(\frac{T_{q} \sin \varphi_{1}-k_{b} \theta_{1}+T_{q} \sin \varphi_{2}+k_{b} \theta_{2}}{Z_{3}}\right) \\
F_{y 2}=-\left(\frac{T_{q} \sin \theta_{1}+k_{b} \varphi_{1}+T_{q} \sin \theta_{2}-k_{b} \varphi_{2}}{Z_{3}}\right) \\
M_{x 2}=T_{q} \sin \theta_{2}-k_{b} \varphi_{2} \\
M_{y 2}=T_{q} \sin \varphi_{2}+k_{b} \theta_{2}
\end{array}\right.
$$

where the misalignment angles $\theta_{1}, \varphi_{1}, \theta_{2}$, and $\varphi_{2}$ can be obtained by the following equations:

$$
\begin{aligned}
& \left\{\begin{array}{l}
\theta_{1}=\sin ^{-1}\left(\frac{\Delta X_{1}}{Z_{3}}\right), \\
\varphi_{1}=\sin ^{-1}\left(\frac{\Delta Y_{1}}{Z_{3}}\right),
\end{array}\right. \\
& \left\{\begin{array}{l}
\theta_{2}=\sin ^{-1}\left(\frac{\Delta X_{2}}{Z_{3}}\right), \\
\varphi_{2}=\sin ^{-1}\left(\frac{\Delta Y_{2}}{Z_{3}}\right) .
\end{array}\right.
\end{aligned}
$$

For angular misalignment,

$$
\left\{\begin{array}{l}
F_{x 2}=\frac{T_{q} \sin \theta_{3}}{Z_{3}}, \\
F_{y 2}=\frac{k_{b} \theta_{3}}{Z_{3}}, \\
M_{x 2}=-k_{b} \theta_{3}, \\
M_{y 2}=T_{q} \sin \theta_{3} .
\end{array}\right.
$$

It can be observed that the reaction forces $F_{x 2}$ and $F_{y 2}$ and the reaction moments $M_{x 2}$ and $M_{y 2}$ are the functions of the angular displacements and lateral translational displacements of misalignment, which are displayed in Figure 6 . The bending spring rate $\left(k_{b}\right)$ and the centre of articulation $\left(Z_{3}\right)$ of the coupling are also displayed in the figure. The parallel and angular misalignment parameters $\left(\Delta X_{1}, \Delta X_{2}, \Delta Y_{1}, \Delta Y_{2}\right.$, and $\left.\theta_{3}\right)$ can be measured using the reverse indicator method. Assuming that $z_{1}$ is the driving side, $(+) T_{q}$ is applied as shown in Figure 6, and the rotation is in the same direction as the applied torque. [44].

As already mentioned in the introduction section, the reaction forces and moments calculated from the Gibbons-Sekhar formula are static loads. For a rotating shaft, the static loads can be decomposed as series dynamic periodic forces which cause vibrations. The dynamic misalignment forces and moments are treated as excitations at the coupling node of the finite element model, and only the $1 x, 2 x, 3 x$, and $4 x$ harmonic components are considered in this study. Assuming that all reacting forces and moments caused by the coupling misalignment are located at node $j$ of the inner rotor, the forces and moments are given as follows $[30,31]$ :

$$
\begin{aligned}
& \mathbf{F}_{c}=\left[\begin{array}{llll}
\mathbf{F}_{c}^{1} & \mathbf{F}_{c}^{2} & \cdots & \mathbf{F}_{c}^{25}
\end{array}\right]^{\mathrm{T}}, \\
& \mathbf{F}_{c}^{1}=\mathbf{F}_{c}^{2}=\cdots=\mathbf{F}_{c}^{j-1}=\mathbf{F}_{c}^{j+1}=\cdots=\mathbf{F}_{c}^{25}=\left[\begin{array}{llll}
0 & 0 & 0 & 0
\end{array}\right], \\
& \mathbf{F}_{c}^{j}=\left[\begin{array}{c}
F_{x 2}\left(\sin \left(\Omega_{j} t\right)+\sin \left(2 \Omega_{j} t\right)+\sin \left(3 \Omega_{j} t\right)+\sin \left(4 \Omega_{j} t\right)\right) \\
F_{y 2}\left(\cos \left(\Omega_{j} t\right)+\cos \left(2 \Omega_{j} t\right)+\cos \left(3 \Omega_{j} t\right)+\cos \left(4 \Omega_{j} t\right)\right) \\
M_{x 2}\left(\sin \left(\Omega_{j} t\right)+\sin \left(2 \Omega_{j} t\right)+\sin \left(3 \Omega_{j} t\right)+\sin \left(4 \Omega_{j} t\right)\right) \\
M_{y 2}\left(\cos \left(\Omega_{j} t\right)+\cos \left(2 \Omega_{j} t\right)+\cos \left(3 \Omega_{j} t\right)+\cos \left(4 \Omega_{j} t\right)\right)
\end{array}\right]^{\mathrm{T}}
\end{aligned}
$$

$\mathbf{F}_{c}^{j}$ is the dynamic reaction forces and moments of the misaligned coupling at node $j . \Omega_{j}$ is the rotation speed of the coupling.

For the dual-rotor system shown in Figure 2, the misalignment occurs in the coupling of the inner rotor. Because the inner rotor is supported by three bearings, the supporting structure can only be an angular misalignment. Suppose that bearing 1 is offset by $\triangle$ millimetre corresponding to a misalignment angle of $\theta_{3}$ degrees which is shown in Figure 7. Therefore, only the effects of the angular misalignment on the spectral characteristics of the dualrotor system will be studied in this paper.

\section{Simulation and Discussions}

In this section, two case studies are performed to investigate the effects of coupling misalignment on the spectral characteristics of the dual-rotor system: (1) the dual-rotor system with only unbalanced excitations; (2) the system with both unbalanced excitations and misaligned excitations. The frequency responses of the two conditions are compared with each other to understand the mechanism of misalignment in the dual-rotor system. Assume that the inner and outer rotors rotate in the reverse direction; the rotation speed of the outer rotor is 1.6 times that of the inner rotor. 


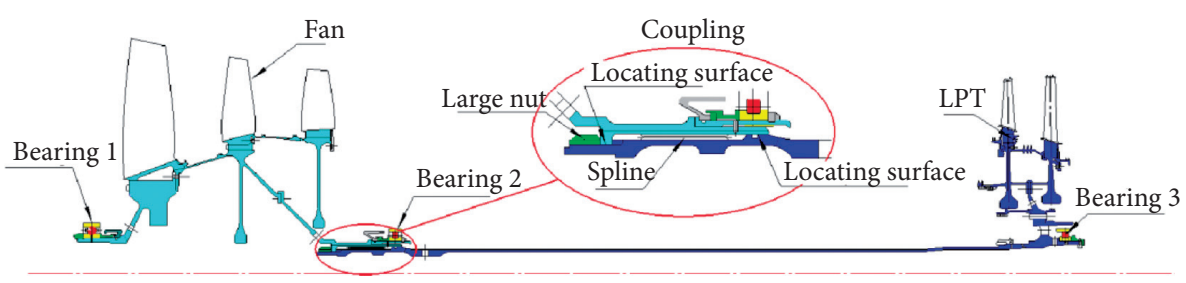

FIgURE 5: The coupling of an aero-engine dual-rotor-bearing system [37].

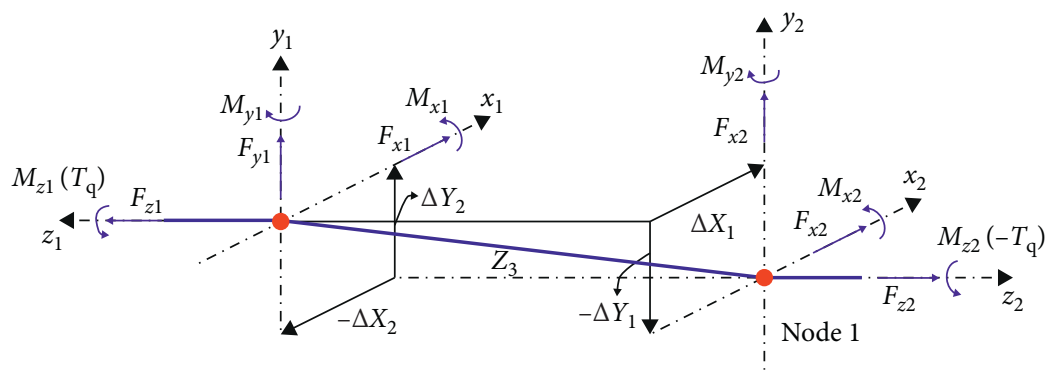

(a)

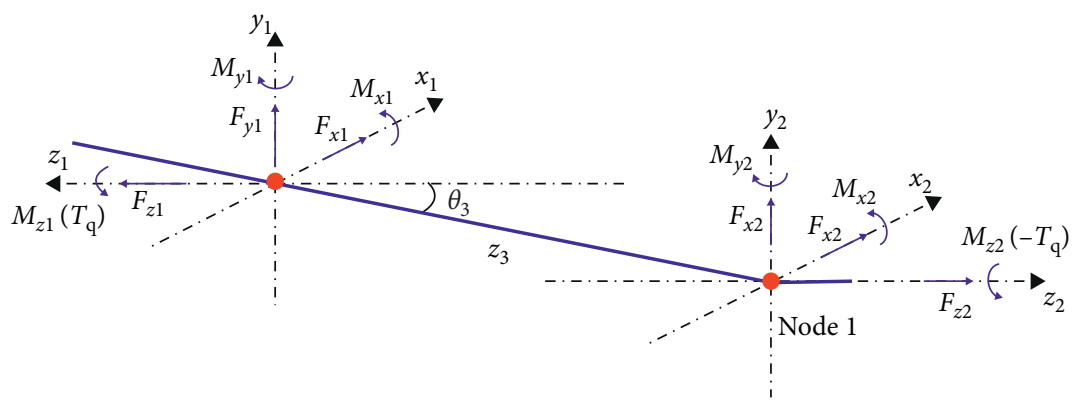

(b)

FIGURE 6: Coupling coordinate system: (a) parallel misalignment and (b) angular misalignment [31].

Regardless of the unbalance moment, assume that only the out-of-balance forces exist at both node 3 (disk 1) of the inner rotor and node 20 (disk 4) of the outer rotor; all reacting forces and moments caused by the coupling misalignment are located at node 6 . And the time domain dynamic response of the system can be obtained by solving the dynamic equation of the dual-rotor system with the Newmark- $\beta$ method. The frequency response of the system can be obtained by the FFT algorithm.

3.1. Frequency Response of Out-of-Balance. A Campbell diagram is a map of natural frequency against rotor rotation speed. It is useful in identifying the forward and backward precession natural frequency components of the rotor system during spectral analysis. The Campbell diagram of the dual-rotor system, plotted against the inner rotor speed, is shown in Figure 8. Shown on the Campbell diagram are the lines giving the correspondence between the natural frequencies plotted on $y$-axis and the rotation speed of the inner rotor plotted on $x$-axis. As the rotor speed increases, each natural frequency pair diverges due to gyroscopic effects. FW in red and BW in black denote the forward and backward natural frequencies, respectively. Therefore, the first-order forward and backward natural frequencies are expressed as 1st BFW and 1st BW, and so on. The first-, second-, third-, and fourth-order vibration of the inner rotor are represented as $1 x, 2 x, 3 x$, and $4 x$, respectively. The firstand second-order vibration of the outer rotor are denoted as $1.6 x$ and $3.2 x$, because the rotating speed of the outer rotor is 1.6 times of that of the inner rotor.

The spectrum diagrams of the dual-rotor system with only unbalance forces are shown in Figures 9 and 10 when the rotation speed of the inner rotor is $1260 \mathrm{rpm}(21 \mathrm{~Hz})$ and that of the outer rotor is $2016 \mathrm{rpm}(33.6 \mathrm{~Hz})$. The figures show the following.

(1) The dominant frequencies of the dual-rotor system are $1 x$ and $1.6 x$ when only unbalanced excitations exist in the system. The amplitude of $1.6 x$ is always greater than that of $1 x$ either in the inner rotor or the outer rotor although the two rotors have the same unbalanced mass. Due to the higher speed of the outer rotor, the unbalanced force of the outer rotor is greater than that of the inner rotor.

(2) The vibration amplitudes in horizontal direction and vertical direction at the same bearing (Figures 9(a) 


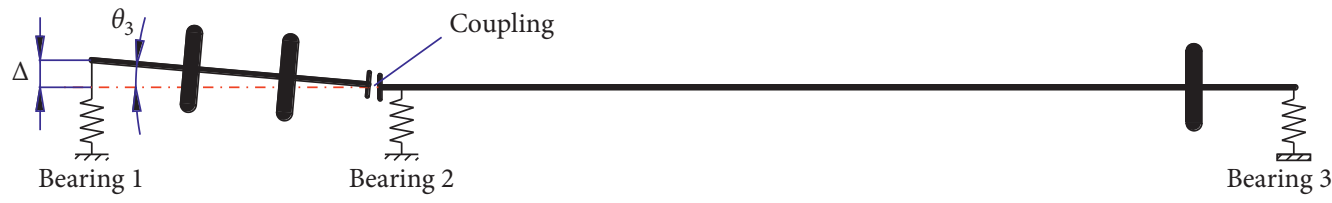

FIGURE 7: Angular misalignment of coupling of the inner rotor.

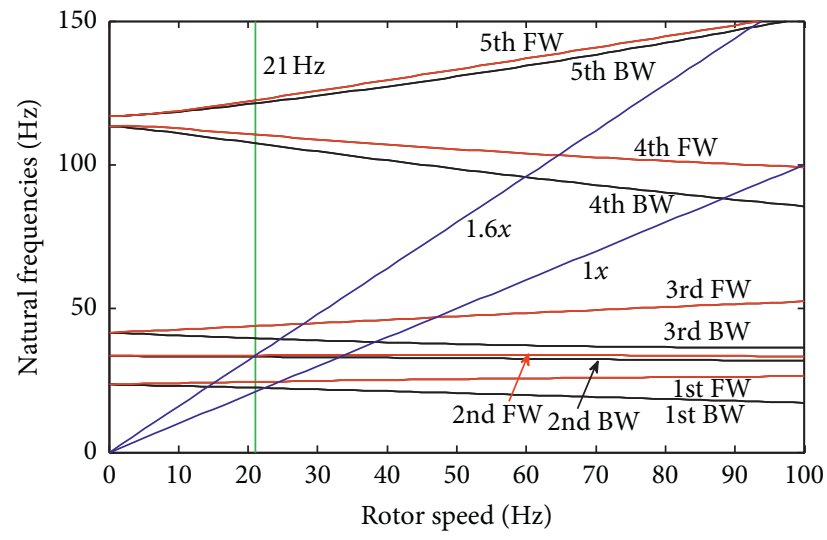

Figure 8: Campbell diagram for the dual-rotor- bearing system.

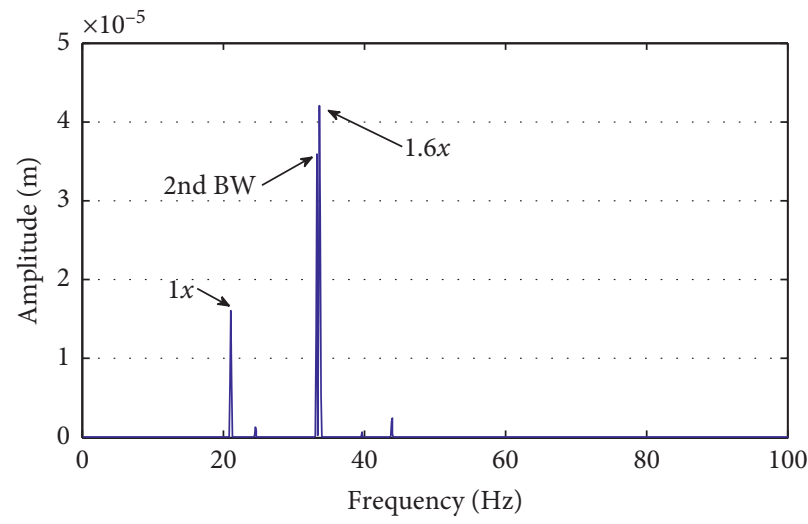

(a)

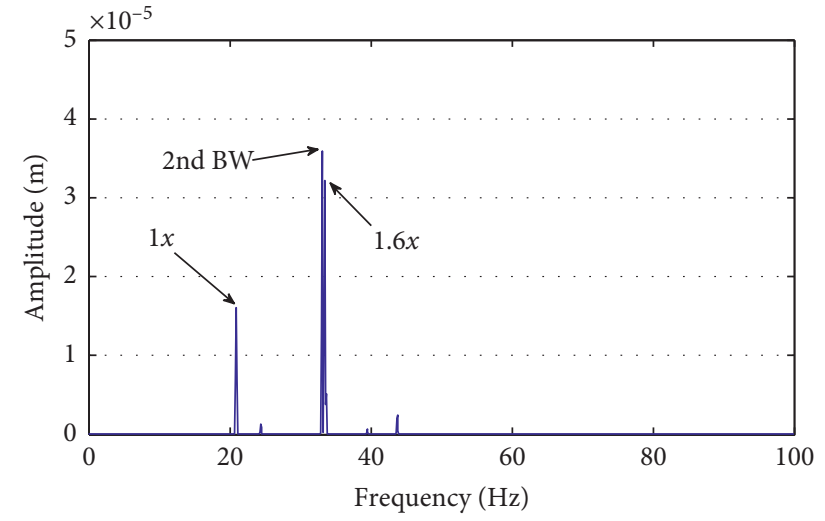

(b)

FIGURE 9: Spectrum of out-of-balance at bearing 1 (inner rotor): (a) horizontal direction and (b) vertical direction.

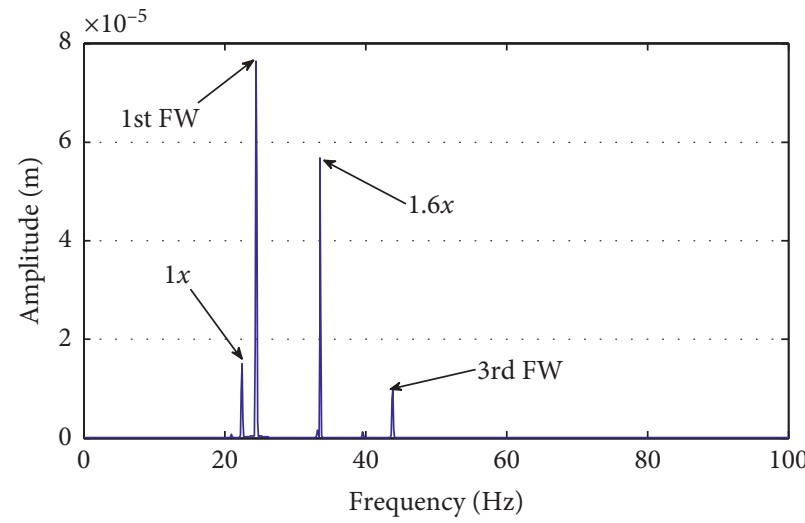

(a)

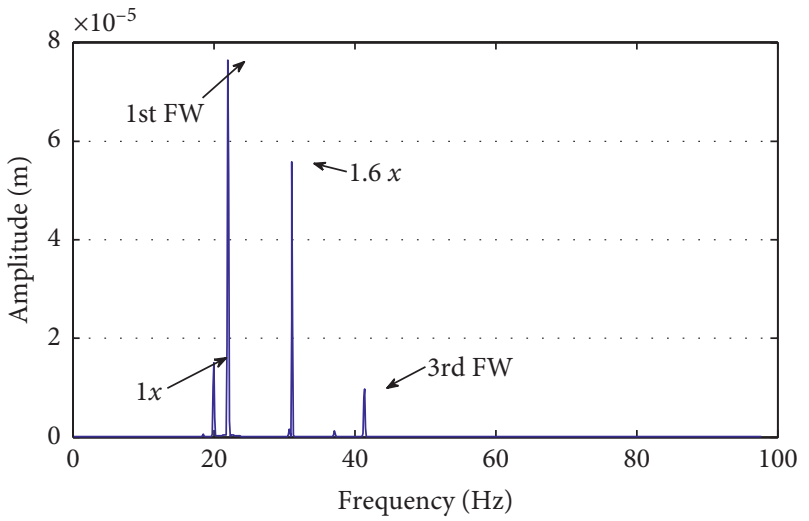

(b)

FIGURE 10: Spectrum of out-of-balance at bearing 4 (outer rotor): (a) horizontal direction and (b) vertical direction. 
and 9(b) and Figures 10(a) and 10(b)) are at the same level, because the stiffness is isotropic. However, it will change obviously when the misalignment reacting force is applied, which will be discussed in Section 3.2. The amplitude of $1.6 x$ in the outer rotor is greater than that in the inner rotor. The vibration attenuated when the unbalance force of the outer rotor passes through the intershaft bearing to the inner rotor.

(3) Resonance occurs at bearing 1 when $1.6 x(33.6 \mathrm{~Hz})$ is close to the 2nd BW natural frequency $(33.18 \mathrm{~Hz})$ of the rotor system, which is shown in Figure 9, so does bearing 4 of the outer rotor, when $1 x(21 \mathrm{~Hz})$ is close to 1st FW $(24.47 \mathrm{~Hz})$, which is shown in Figure 10. This is different from the Jeffcott rotor whose unbalance force can only arouse the forward precession natural frequency. This is caused by the different rotation directions of the inner and outer rotors. The unbalanced forces on the outer rotor play a dominant role, which caused the outer rotor whirl in the forward precession while the inner rotor is in the backward precession.

3.2. Frequency Response of Misalignment. In order to analyse the influence of misalignment on the spectrum characteristics of the dual-rotor system, it is assumed that the working conditions of the rotor system are exactly the same as the unbalanced conditions (as described in Section 3.1) except for the additional misalignment forces and moments. Bearing 1 is assumed to be up $0.3 \mathrm{~mm}(\Delta=0.3 \mathrm{~mm})$ in the vertical direction, shown in Figure 7, and the inner rotor produces a misalignment angle of $0.21^{\circ}$. The reacting forces and moments are calculated and substituted into the dynamic equations of the system. The misalignment responses of the rotor system are obtained by solving the dynamic equation. The spectra of bearing 1 and bearing 4 are shown in Figures 11 and 12. The spectra of bearing 2 and bearing 3 are not separately listed here because they are similar to that of bearing 1 and bearing 4 . The spectrum of bearing 5 is not shown separately because the vibration signal of the intershaft bearing cannot be verified by a direct test measurement.

Comparing Figures 11 and 12 with Figures 9 and 10, the following is shown:

(1) The vibration spectrum of misalignment is more complicated than that of unbalance. Both the inner and outer rotors appear frequency components dominated by $1 x, 1.6 x$, and $2 x$. In addition, $3 x, 4 x$, and multiple forward and backward precession natural frequencies are also excited by the misalignment. In particular, both the 3rd FW and 3rd BW natural frequencies of the rotor are excited in the vicinity of $2 x$ frequency.

(2) Misalignment intensifies the vibration in the misalignment direction. Comparing Figures 11(a) and 11(b) indicates that the vibration amplitude of bearing 1 in the vertical direction is significantly higher than that in the horizontal direction, although the stiffness of bearing 1 is isotropic. It is due to that the intentional misalignment was set in the vertical direction. The force of misalignment is directional, which does not rotate with the rotor. However, the unbalance causes a rotating force, which is a onceper-revolution fault. The direction of the unbalanced force will rotate synchronously with the rotor. Thus, the huge gap between the two directions will not appear in the unbalanced response. This phenomenon is not obvious on bearing 4 of the outer rotor by comparing Figures 12(a) and 12(b), because bearing 4 is far from the misaligned coupling.

(3) Note that the further away from the misalignment coupling, the less affected by misalignment. Misalignment increases the vibration level of both the inner and outer rotor, but the most obvious change is in the direction of misalignment near the misaligned coupling. The maximum vibration amplitude of bearing 1 is higher than that of bearing 4 under the condition of misalignment (Figures 11 and 12), because bearing 4 is further away from the misaligned coupling. However, as shown in Figures 9 and 10 in Section 3.1, the maximum vibration amplitude of bearing 1 is lower than that of bearing 4 when only an unbalanced force excites the system. The vibration of bearing 3 also proves this phenomenon. Due to the similar situation, the spectral of bearing 3 is not listed repetitively.

The radar chart shown in Figure 13 is plotted in order to further illustrate the effects of misalignment on different bearings and different harmonic frequency components. As shown in Figure 13, each spoke represents the vibration direction of a bearing. The length of a spoke represents the amplitude of a frequency component. $\mathrm{B} 1 \mathrm{H}$ represents the horizontal direction of bearing 1 and B1V stands for the vertical direction of bearing 1 , and so on. The figures show the following:

(1) Although bearing 2 is closest to the misaligned coupling, the maximum amplitude of the four bearings does not occur at the misalignment direction of bearing 2 . Instead, the maximum amplitude occurs in the vertical direction of bearing 1 (B1V) because the inner rotor is a redundantly support rotor. The vibration of bearing 2 is suppressed because bearing 2 and bearing 3 make the inner rotor a stable structure.

(2) Although the stiffness of the four bearings is isotropic. The vibration amplitudes in the vertical direction are greater than that of the horizontal direction in both bearing 1 and bearing 2, which are near the misaligned coupling. However, bearing 3 and bearing 4, away from the coupling, show the opposite vibration level.

(3) Although the amplitudes of the four harmonic components of the additional force are the same, the amplitudes of the $2 x$ component change most 


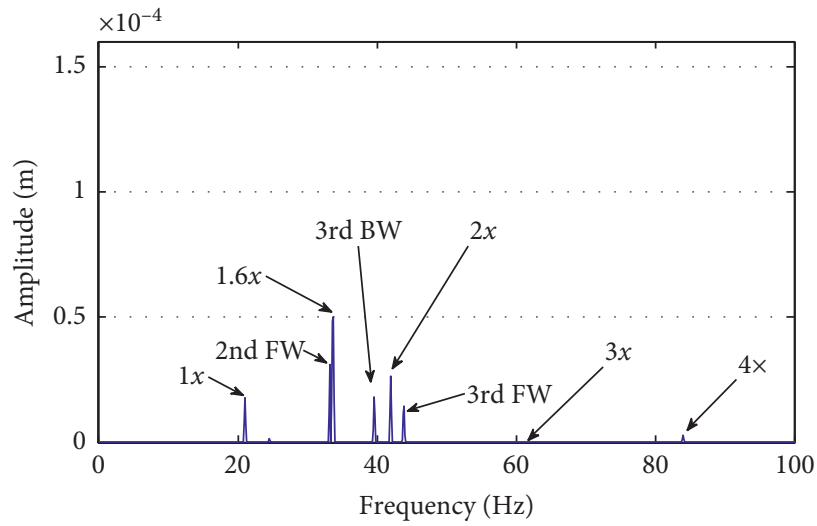

(a)

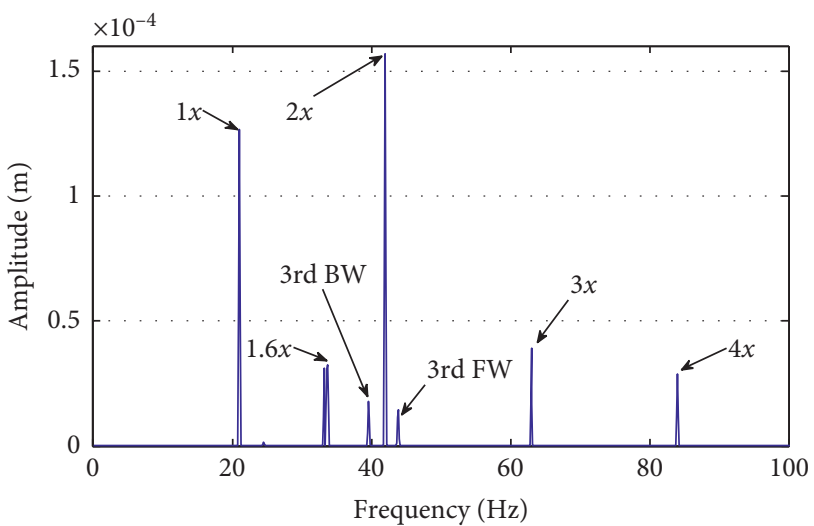

(b)

FIGURE 11: Spectrum of misalignment at bearing 1 (inner rotor): (a) horizontal direction and (b) vertical direction.

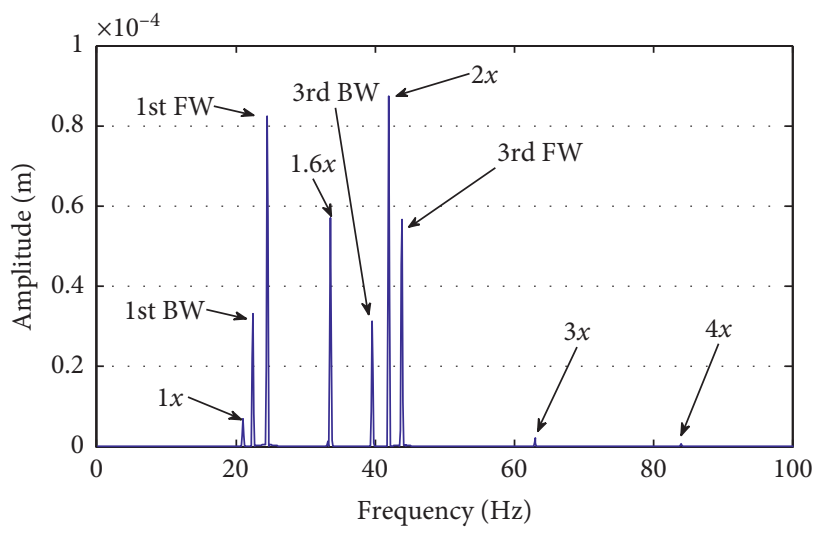

(a)

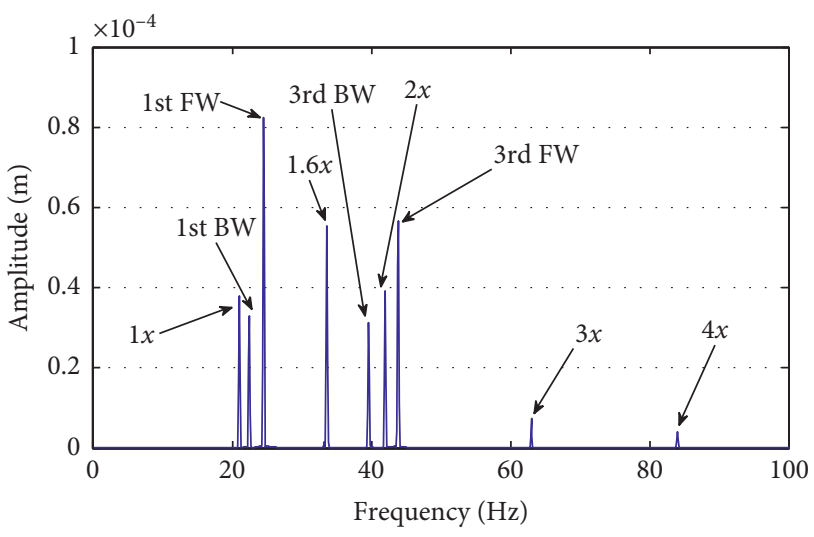

(b)

FIGURE 12: Spectrum of misalignment at bearing 4 (outer rotor): (a) horizontal direction and (b) vertical direction.

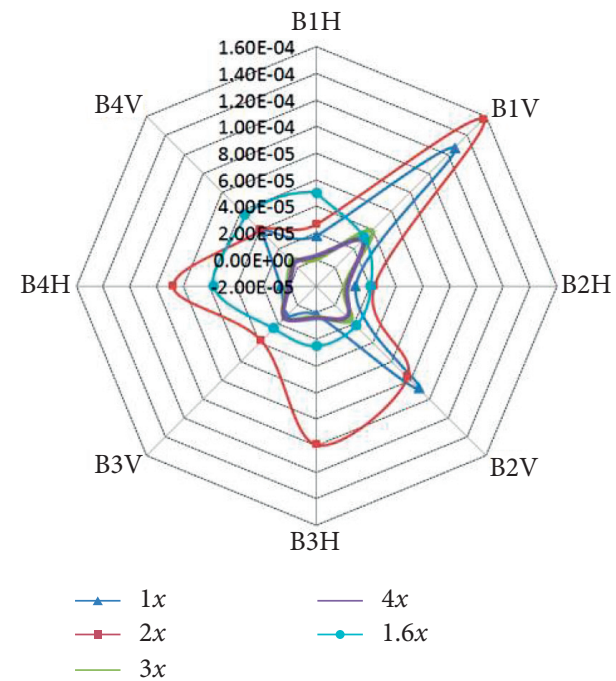

FIGURE 13: Radar chart of harmonic frequencies at the four bearings. significantly. The frequencies of $3 x$ and $4 x$ change a little. But that's not always the case when a resonance occurs, which will be explained in Section 3.3. The $1.6 x$ radar diagram is nearly circular, indicating that the first-order component of the vibration of the outer rotor $(1.6 x)$ is almost unaffected by the misalignment of the inner rotor.

The effects of misalignment on rotor frequency characteristics have been qualitatively analysed. The quantitative influence of different degrees of misalignment on the harmonic frequencies of the system will be analysed too. Assuming that bearing 1 is raised by $0.1 \mathrm{~mm}, 0.2 \mathrm{~mm}$, $0.3 \mathrm{~mm}, 0.4 \mathrm{~mm}$, and $0.5 \mathrm{~mm}$, respectively, the variation trend of the vertical amplitude of bearing 1 with the misalignment value is obtained, which is shown in Figure 14. It can be seen that the amplitude of all harmonic components increases with the increasing of the misalignment angle, but the frequency of $1 x$ and $2 x$ increases more obvious than $3 x$ and $4 x$. 


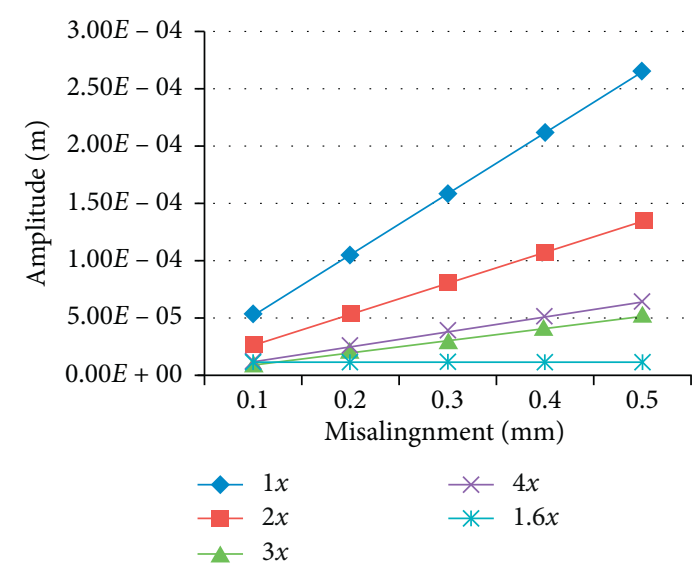

FIGURE 14: Amplitude of harmonic frequencies altering with misalignment.

3.3. Harmonic Resonance. As described in Section 3.1, resonance occurs when the frequency of the unbalance force is close to a natural frequency. Resonance occurs in the misalignment response too. Any harmonic frequency of the misalignment response may be coincided with a natural frequency of the system, which causes resonance. The resonance of bearing 1 in the vertical direction at different rotation speeds is shown in Figures 15-17.

The spectrum of bearing 1 is shown in Figure 15 when the inner rotor speeds are $11.5 \mathrm{~Hz}$ and $13 \mathrm{~Hz}$. It can be found that the amplitude of $3 x$ at $11.5 \mathrm{~Hz}$ is almost six times that of $3 x$ at $13 \mathrm{~Hz}$ although the spectrum components are the same at the two speeds. At the rotation speed of $11.5 \mathrm{~Hz}$, the resonance occurs because the 2 nd FW $(33.52 \mathrm{~Hz})$ is close to $3 x(34.5 \mathrm{~Hz})$. However, the amplitude decreases significantly when the rotation speed is increased to $13 \mathrm{~Hz}$. Similarly, the frequency $2 x$ coincides with the 2 nd FW natural frequency when the rotation speed is $17 \mathrm{~Hz}$. The resonance occurs which is shown in Figure 16(a). However, there is no resonance when the rotation speed is increased to $19 \mathrm{~Hz}$, which is shown in Figure 16(b). The frequency $1 x$ coincides with the 2 ndBW natural frequency when the rotation speed is $32 \mathrm{~Hz}$. Resonance occurs which is shown in Figure 17(a). No resonance occurs when the speed is reduced to $30 \mathrm{~Hz}$, which is shown in Figure 17(b).

From the above analysis, it can be known that all harmonic frequencies excited by misaligned coupling, $1 x, 2 x, 3 x$ and $4 x$, may cause resonance. Even the weak frequencies, like $3 x$ and $4 x$, can cause severe vibration when resonance occurs. Therefore, it is necessary to consider the harmonic resonance of misaligned coupling in the design of a dualrotor system.

3.4. Orbit Analysis. An orbit is a powerful diagnostic tool which can provide important and useful information about the dynamic motion of the rotor. The orbits of the dual-rotor system with different degree misalignment are shown in Figures 18-20. According to the previous analysis, the misalignment has the greatest impact on bearing 1 and the weakest impact on bearing 4 . Therefore, bearing 1 and bearing 4 are taken as the research object to analyse the influence of misalignment on orbits. The influence of misalignment on the orbit of bearing 2 and bearing 3 is between bearing 4 and bearing 1 and is no longer listed separately. The orbit plots of rotor without and with misalignment are shown in Figures 18 and 19 when the inner rotor speed is $19 \mathrm{~Hz}$ and the outer rotor speed is $30.4 \mathrm{~Hz}$. The orbit plots of rotor with different degree misalignment are shown in Figure 20 when the inner rotor speed is $11 \mathrm{~Hz}$ and the outer rotor speed is $17.6 \mathrm{~Hz}$. A comparative analysis of the above figures shows the following:

(1) The orbits of bearing 1 and bearing 4 are both in the shape of rings when only unbalanced excitation exists, as shown in Figure 18. The orbits are multilooped rings rather than perfect circle because the two rotors of the dual-rotor system running at different speeds and the vibration frequency components are more complex than the single $1 x$ frequency.

(2) The orbits of bearing 1 tend to collapse toward a straight line for the angular misalignment along the $y$-direction. Nikolajsens [24] also reported the same phenomenon of misalignment. Figures 19(a) and 20(a) show that the shape of orbits will change with the rotation speed when the misalignment is at the same degree. However, Figures 18(b) and 19(b) show that the orbit of bearing 4 has little change due to the weak influence of misalignment.

(3) Figure 20 shows that the shape of orbits change with the degree of misalignment. And the orbit diverges into a reticulated diamond shape from a straight line shape as the increase of misalignment (from $0.1 \mathrm{~mm}$ to $0.3 \mathrm{~mm}$ ).

\section{Experimental Study of the Rotor System with Coupling Misalignment}

A dual-rotor test rig is built in order to verify the simulation results. Experiments were performed on the test rig under different intentional misalignment conditions.

4.1. Test Rig Configuration. The configuration of the test rig is shown in Figure 21. The test rig mainly consists of the following four parts: (1) a pair of rotors, an inner rotor and an outer rotor, and they rotate in different speed and reverse direction; (2) a pair of frequency-variable motors: the inner motor and outer motor independently drive the inner rotor and outer rotor; (3) five identical disks: disk 1, disk 2, and disk 3 , are mounted on the inner rotor and disk 4 and disk 5 are mounted on the outer rotor; (4) five bearings: the inner rotor is supported by three bearings, namely, bearing 1 , bearing 2 , and bearing 3 . The outer rotor is supported by bearing 4 and bearing 5 (intershaft bearing). The bearing types are presented in Table 5 . The high supporting stiffness of the five bearings makes the critical speed of the dual-rotor system exceed the rated speeds of the two motors. Therefore, five squirrel cages are used to support the bearings to reduce the supporting stiffness. Bearing 1 is padded up by inserting 


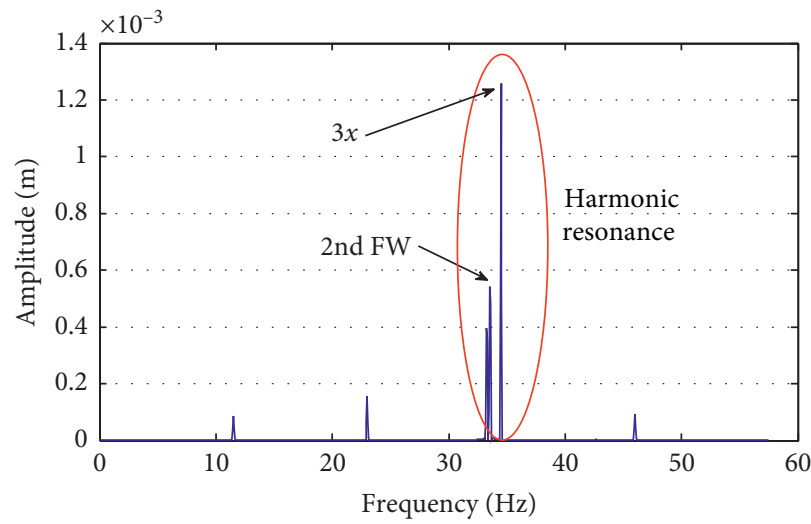

(a)

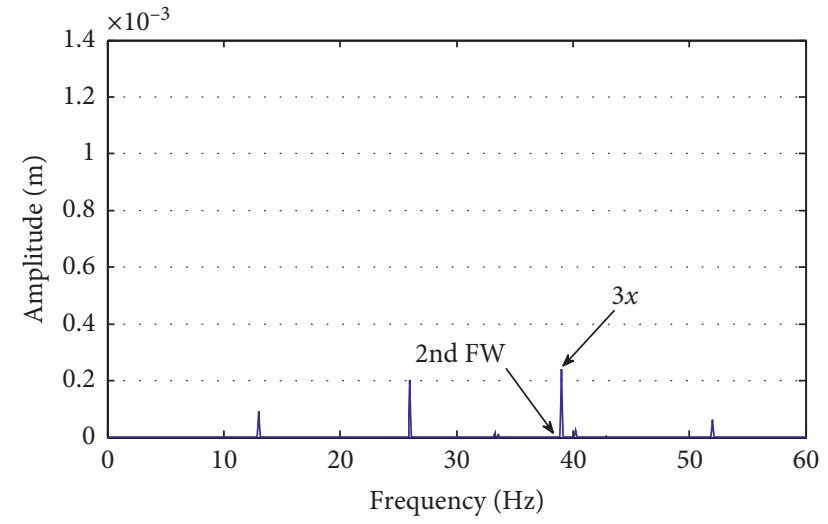

(b)

FIGURE 15: Resonance of $3 x$ in the vertical direction of bearing 1: (a) $11.5 \mathrm{~Hz}$ and (b) $13 \mathrm{~Hz}$.

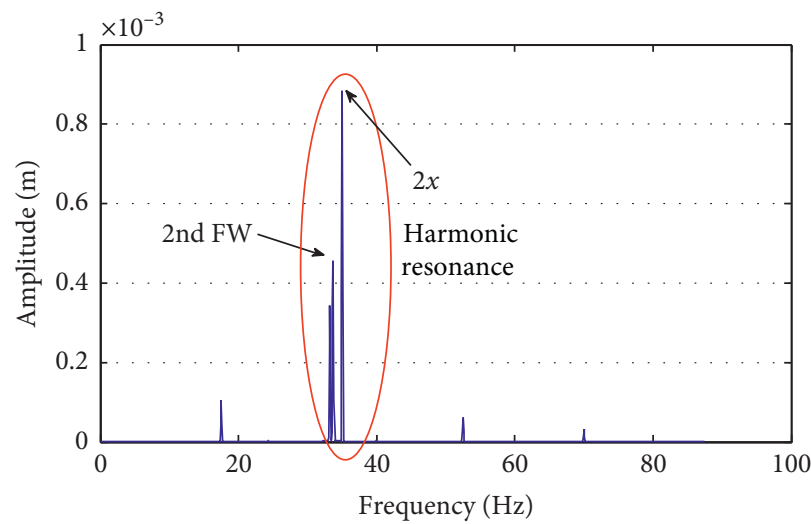

(a)

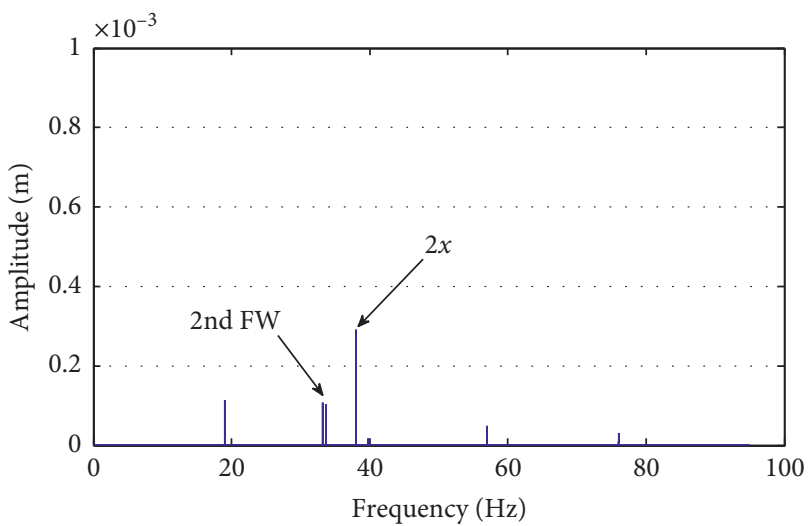

(b)

Figure 16: Resonance of $2 x$ in the vertical direction of bearing1: (a) $17 \mathrm{~Hz}$ and (b) $19 \mathrm{~Hz}$.

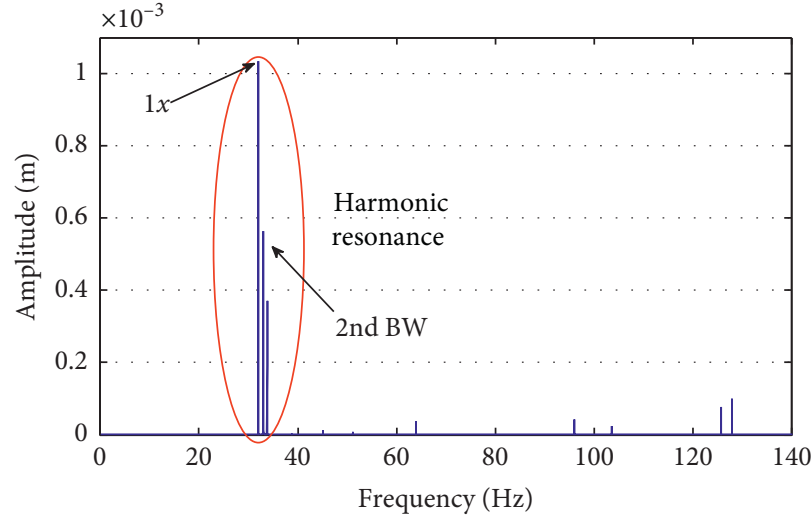

(a)

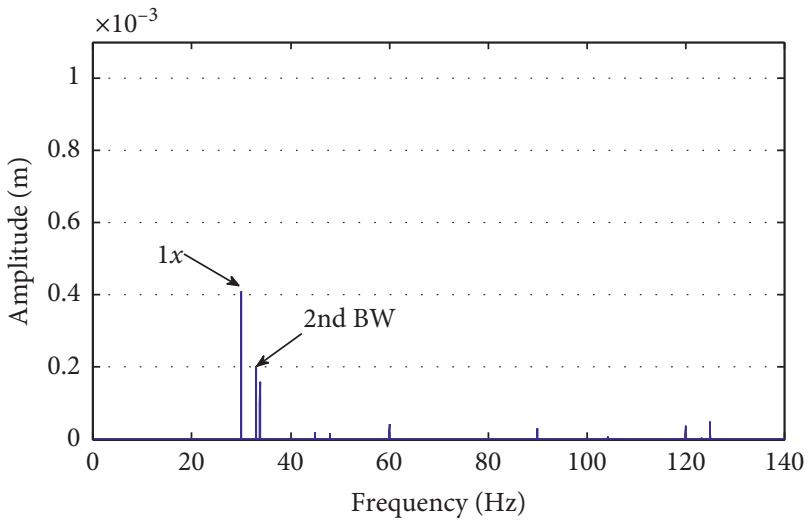

(b)

FIGURE 17: Resonance of $1 x$ in the vertical direction of bearing 1: (a) $32 \mathrm{~Hz}$ and (b) $30 \mathrm{~Hz}$.

gaskets (shown in Figure 22) under the bearing pedestal to produce intentional misalignment.

Vibration data are acquired from the four bearing pedestals by a B \& K data collector. Both horizontal and vertical vibrations of the bearing pedestals are measured by accelerometers. The acceleration signal collected by the sensor is firstly converted into a displacement signal by double integration, and then the FFT algorithm is applied on 


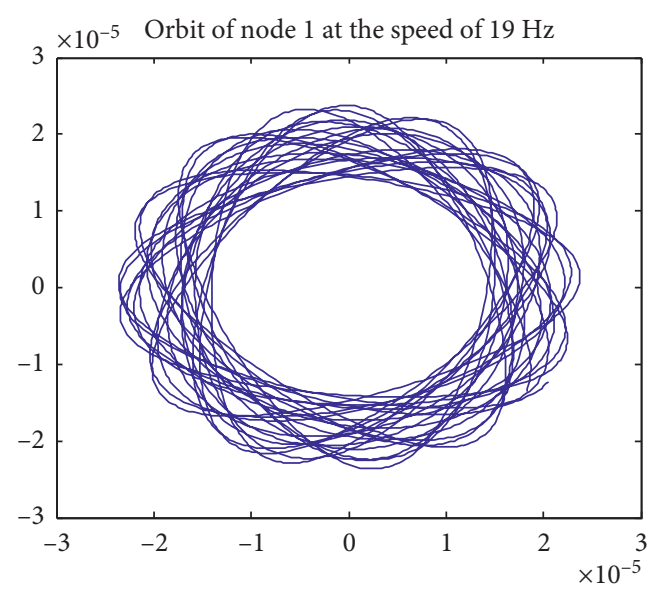

(a)

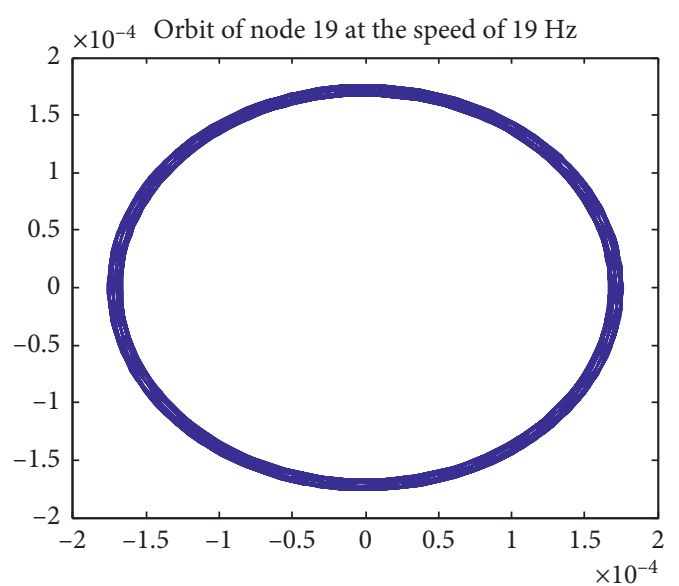

(b)

FIGURE 18: Orbits of bearings without misalignment $\left(\Delta=0 \mathrm{~mm}, \Omega_{1}=19 \mathrm{~Hz}, \Delta_{2}=30.4 \mathrm{~Hz}\right)$ : (a) bearing 1 and (b) bearing 4 .

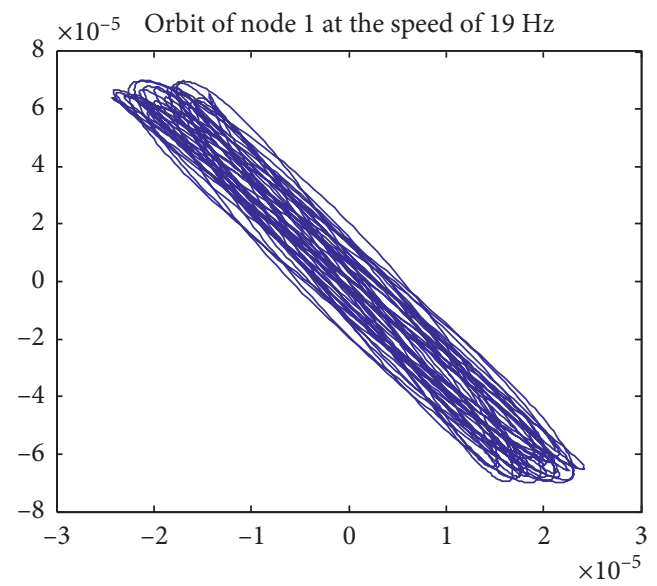

(a)

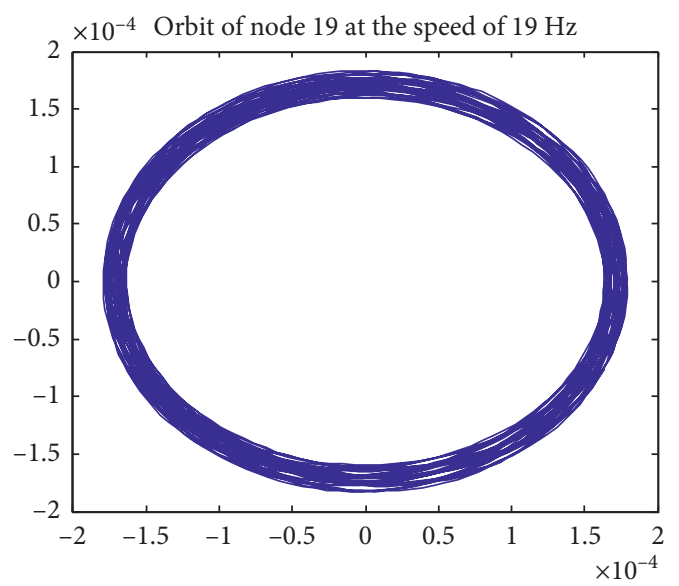

(b)

Figure 19: Orbits of bearings with misalignment $(\Delta=0.1 \mathrm{~mm}, \Omega 1=19 \mathrm{~Hz}, \Omega 2=30.4 \mathrm{~Hz}$ ): (a) bearing 1 and (b) bearing 4 .

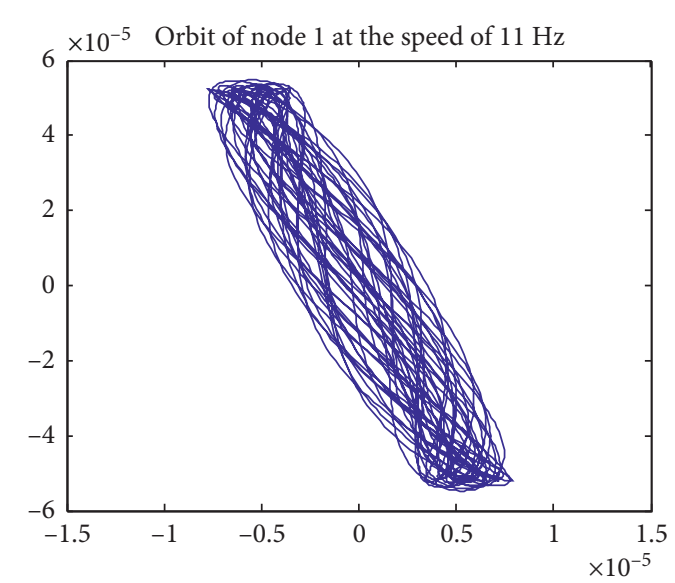

(a)

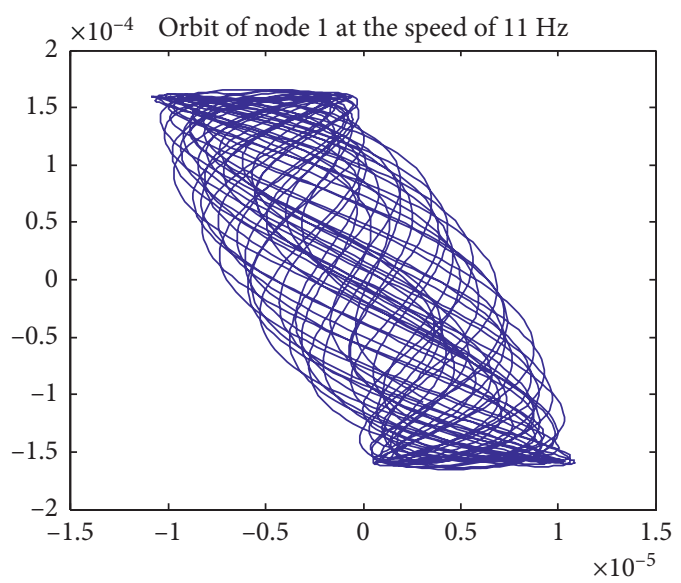

(b)

Figure 20: Orbits of bearing 1 at different misalignment $\left(\Omega_{1}=11 \mathrm{~Hz}, \Omega_{2}=17.6 \mathrm{~Hz}\right.$ ): (a) $\Delta=0.1 \mathrm{~mm}$ and (b) $\Delta=0.3 \mathrm{~mm}$. 


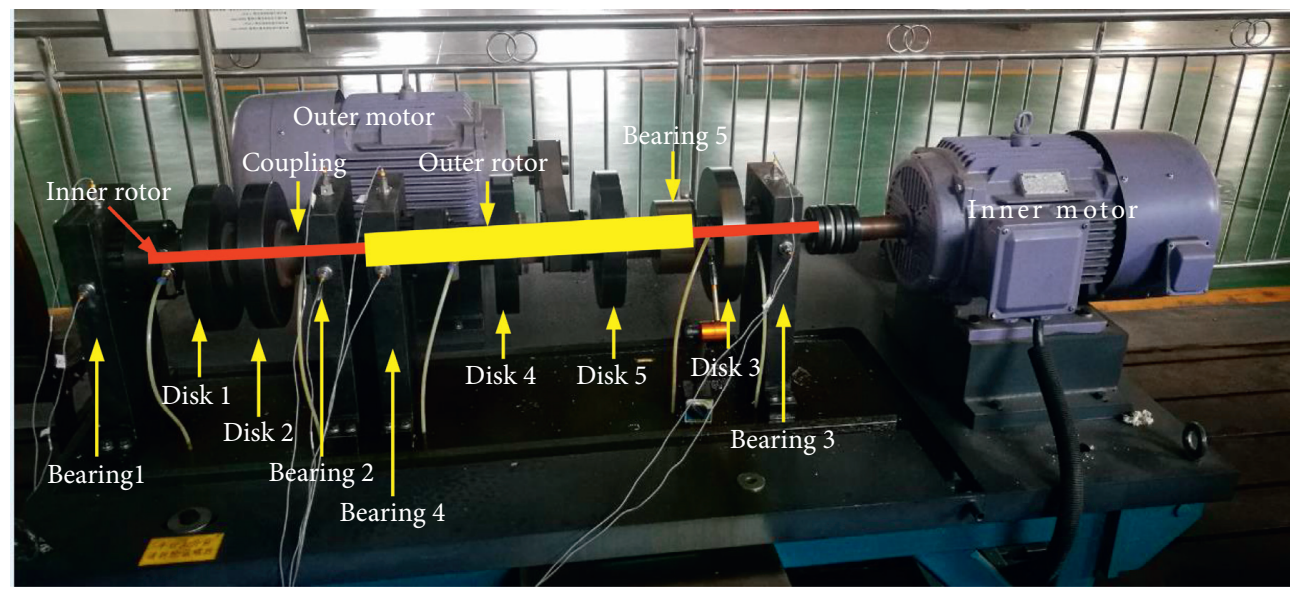

Figure 21: Experimental setup of the dual-rotor system with five supports.

TABLE 5: Bearing type of the test rig.

\begin{tabular}{lc}
\hline Bearing & Types \\
\hline Bearing 1 & NU1013 \\
Bearing 2 & $7013 \mathrm{AC}$ \\
Bearing 3 & NU1013 \\
Bearing 4 & $7013 \mathrm{AC}$ \\
Bearing 5 & NU1013 \\
\hline
\end{tabular}

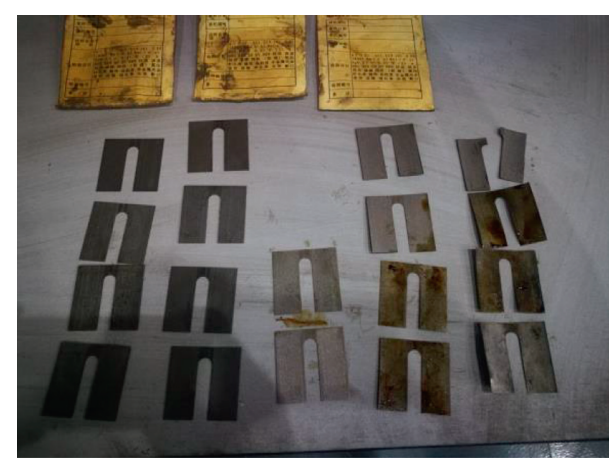

Figure 22: Gaskets of different thickness.

the displacement signal to obtain the spectrum. Because the low-frequency noise will be generated in this process, a highpass filter is adopted to filter low-frequency noise signals below $10 \mathrm{~Hz}$.

4.2. Experimental Results and Discussions. Three test plans are performed to verify the simulation results.

The first experiment aims to confirm the spectral components of the misaligned dual-rotor system. When the spacer thickness is $0.3 \mathrm{~mm}$ and the rotation speed of the inner rotor is $21 \mathrm{~Hz}$, the spectra of bearing 1 and bearing 4 are shown in Figures 23 and 24. The figures indicate the following.

(1) The misalignment excited the frequencies of $1 x, 2 x$, $3 x, 4 x$, and $1.6 x$ and several natural frequencies, which are consistent with the theoretical analysis as shown in Figures 11 and 12. It should be pointed out that the second-order component of vibration of the outer rotor (denoted by $3.2 x$ ) appeared in the experimental spectrum. It does not appear in simulation. It may be caused by the misalignment between the inner and outer rotor. Further study about $3.2 x$ will be conducted in the future.

(2) Comparing the vibration amplitude of both directions (Figures 23(a) and 23(b) and Figures 24(a) and $24(b))$ of the same bearing, it can be seen that the vibration amplitude of the vertical direction is larger than that of the horizontal direction, although the actual stiffness of bearing pedestal in the vertical direction is greater than that in the horizontal direction. This phenomenon confirms the simulation results that misalignment will intensify the vibration in the misalignment direction.

(3) The vibration level of bearing 1 is higher than that of bearing 4 in both horizontal and vertical directions, which can be illustrated by comparing Figures 23(a) and 24(a) and Figures 23(b) and 24(b). The experiment results also agree with the simulation results shown in Section 3.2.

The second experiment is conducted to check the effects of different degrees of misalignment on the amplitude of different frequency components. Gaskets with thickness of $0.2 \mathrm{~mm}, 0.3 \mathrm{~mm}, 0.4 \mathrm{~mm}, 0.5 \mathrm{~mm}$, and $0.6 \mathrm{~mm}$, shown in Figure 22, are customized to produce different misalignment angles. Because the $0.6 \mathrm{~mm}$ gasket is too thick, it is easy to cause bearing 1 and bearing 2 to heat and smoke. Therefore, the $0.6 \mathrm{~mm}$ gasket was not used for the experiment. The $0.1 \mathrm{~mm}$ thick gasket is too thin to be processed. Therefore, only $0.2 \mathrm{~mm}, 0.3 \mathrm{~mm}, 0.4 \mathrm{~mm}$, and $0.5 \mathrm{~mm}$ gaskets are used for the experiments.

The spectra corresponding to different spacer thicknesses are tested at the same speed $\left(\Omega_{1}=21 \mathrm{~Hz}\right.$, $\Omega_{2}=33.6 \mathrm{~Hz}$ ). Two sets of tests are performed on each gasket, recording the amplitude of each frequency component. Then the amplitude of each frequency is averaged, and the experimental results are finally obtained as shown in 


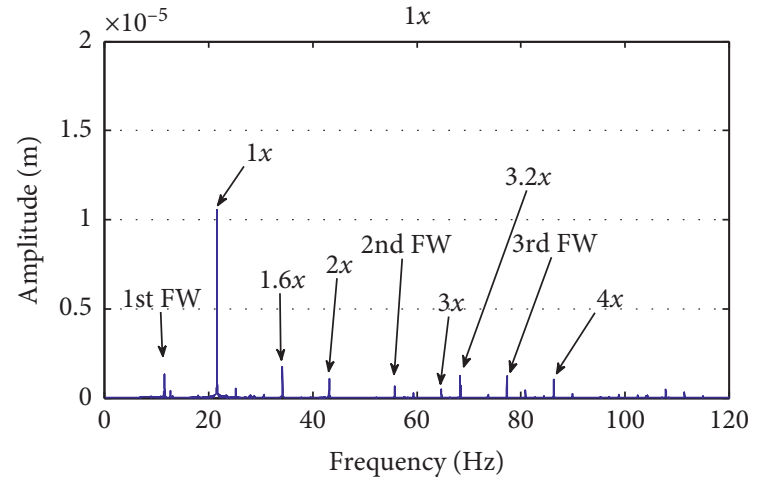

(a)

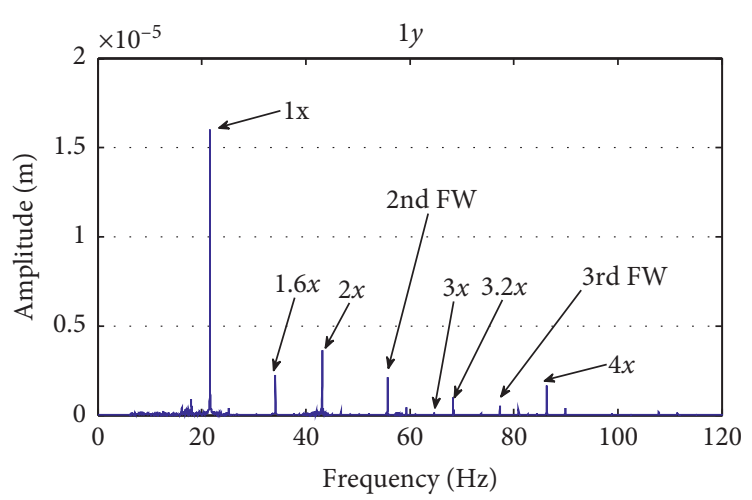

(b)

FIGURE 23: Spectrum of misalignment and unbalance at bearing 1, $\Omega_{1}=21 \mathrm{~Hz}$ : (a) horizontal direction and (b) vertical direction.

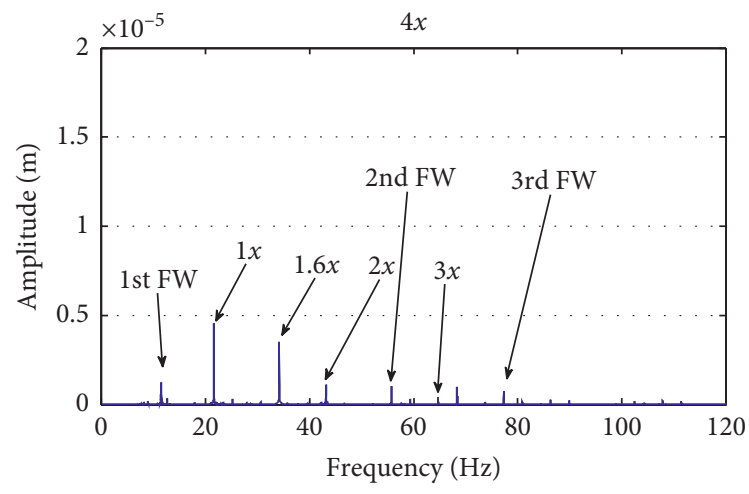

(a)

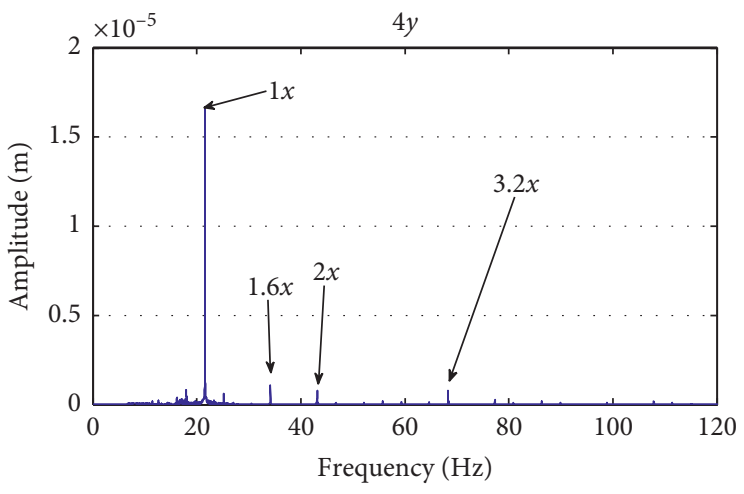

(b)

FIGURE 24: Spectrum of misalignment and unbalance in bearing 4, $\Omega_{1}=21 \mathrm{~Hz}$ : (a) horizontal direction and (b) vertical direction.

Figure 25. The test results confirm the simulation results shown in Figure 14 that the amplitude of each harmonic component increases with the degree of misalignment. This result is not completely consistent with Dewell's [32] research results, which showed that $1 x$ does not change with the degree of misalignment. This may be due to the different structure of the coupling. Instead of a spline coupling, a disk coupling is the research object in his study.

The third experiment is designed to verify the harmonic resonance caused by misalignment. The vibration of bearing 1 and bearing 4 at two rotation speeds is measured, as shown in Figures 26 and 27. It can be clearly seen from the two figures that the harmonic resonance occurs when the rotation speed of the inner rotor is $18 \mathrm{~Hz}$, because $3 x$ is close to 2nd FW. This is not the case at $11 \mathrm{~Hz}$. The test is consistent with the theoretical analysis too.

Due to the limitation of experimental conditions, the orbits of the rotor cannot be measured directly. The orbits of the rotor are replaced by the orbits of bearings to analyse the influence of misalignment. The orbits of bearings are plotted by the vibration displacement of bearing pedestals in horizontal and vertical directions. Figure 28 shows the orbits without misalignment when the speeds of the inner rotor and outer rotor are $1300 \mathrm{rpm}$ $(21.6 \mathrm{~Hz})$ and $2080 \mathrm{rpm}(34.6 \mathrm{~Hz})$. The orbits with misalignment

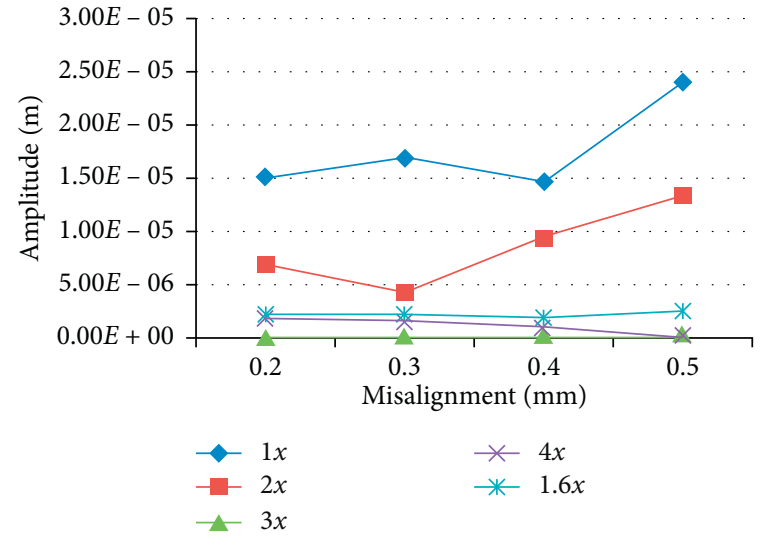

FIgURE 25: Amplitude of harmonic frequencies altering with misalignment value (test results).

$(\Delta=0.2 \mathrm{~mm})$ are shown in Figure 29. By comparing the two figures, it can be seen that the orbit of bearing 1 tend to collapse toward a straight line because of the reacting forces and moments caused by the misalignment. Figure 30 shows that the orbits diverge toward a shape of reticulated diamond with the severity of misalignment (from $0.2 \mathrm{~mm}$ to $0.4 \mathrm{~mm}$ ). The experimental results confirm the simulation results very well. 


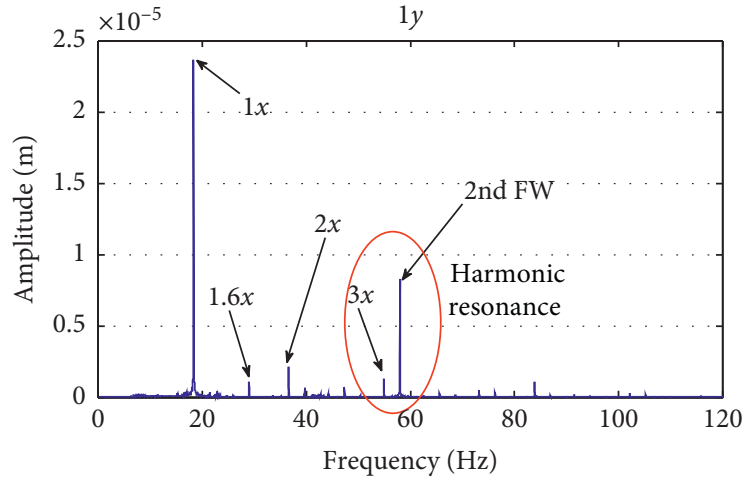

(a)

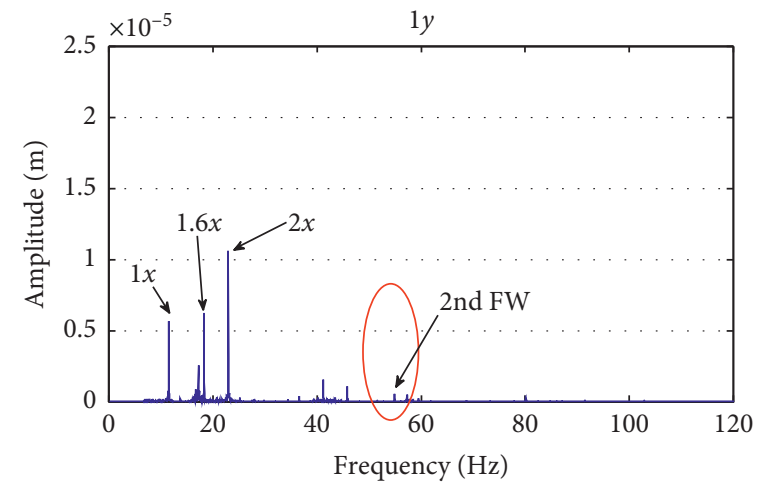

(b)

FIGURE 26: Spectrum of misalignment and unbalance at bearing 1: (a) horizontal $\left(\Omega_{1}=18 \mathrm{~Hz}, \Omega_{2}=28.8 \mathrm{~Hz}\right)$ and (b) horizontal $\left(\Omega_{1}=11 \mathrm{~Hz}\right.$, $\left.\Omega_{2}=17.6 \mathrm{~Hz}\right)$.

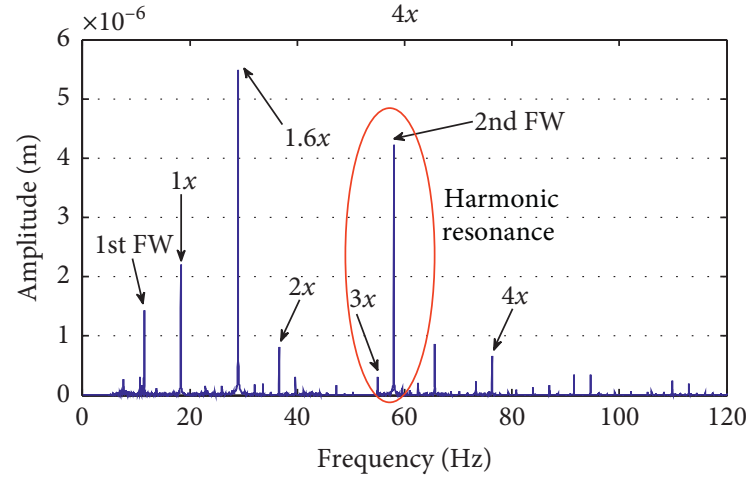

(a)

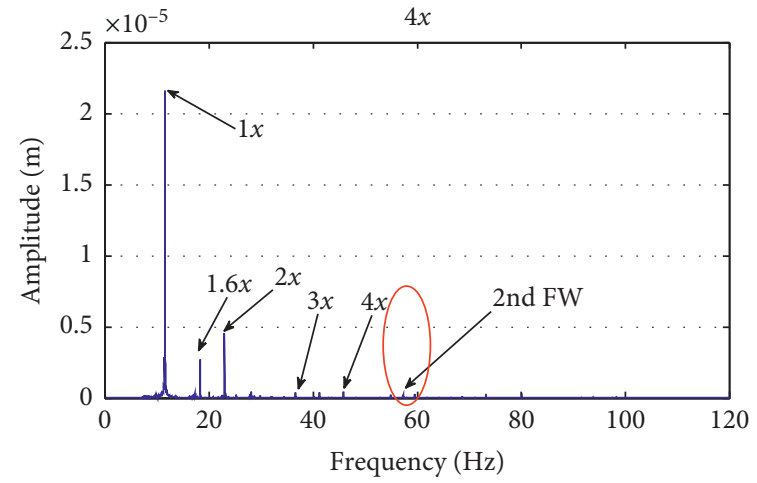

(b)

FIGURE 27: Spectrum of misalignment and unbalance in bearing 4. (a) horizontal $\left(\Omega_{1}=18 \mathrm{~Hz}, \Omega_{2}=28.8 \mathrm{~Hz}\right)$ and (b) horizontal $\left(\Omega_{1}=11 \mathrm{~Hz}\right.$, $\Omega_{2}=17.6 \mathrm{~Hz}$.

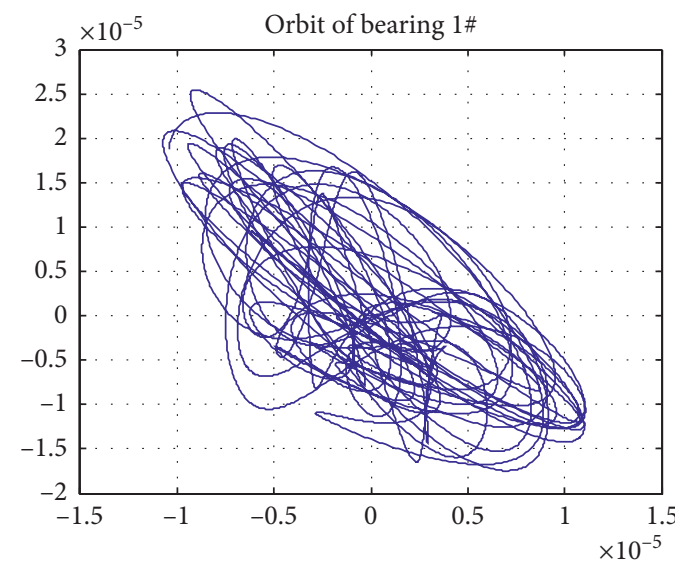

(a)

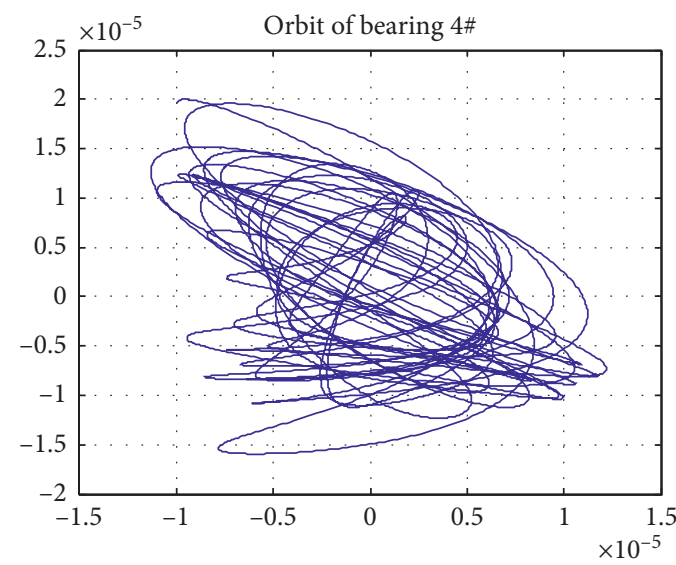

(b)

FIGURE 28: Orbits of bearing without misalignment $\left(\Delta=0 \mathrm{~mm}, \Omega_{1}=21.6 \mathrm{~Hz}, \Omega_{2}=34.6 \mathrm{~Hz}\right.$ ): (a) bearing 1 and (b) bearing 4 . 


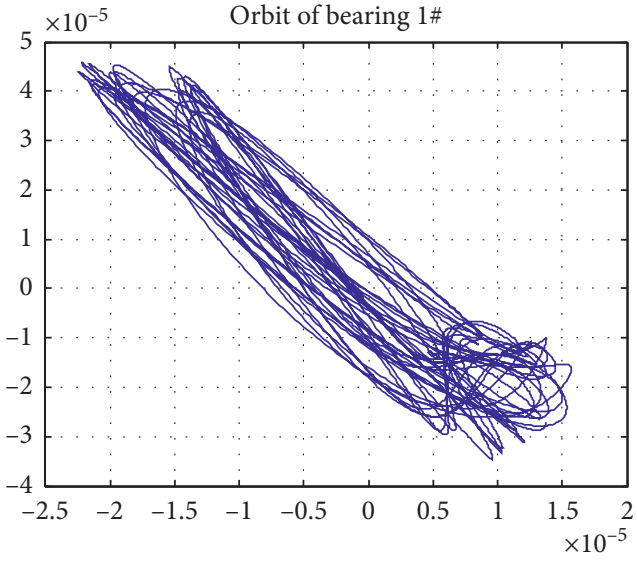

(a)

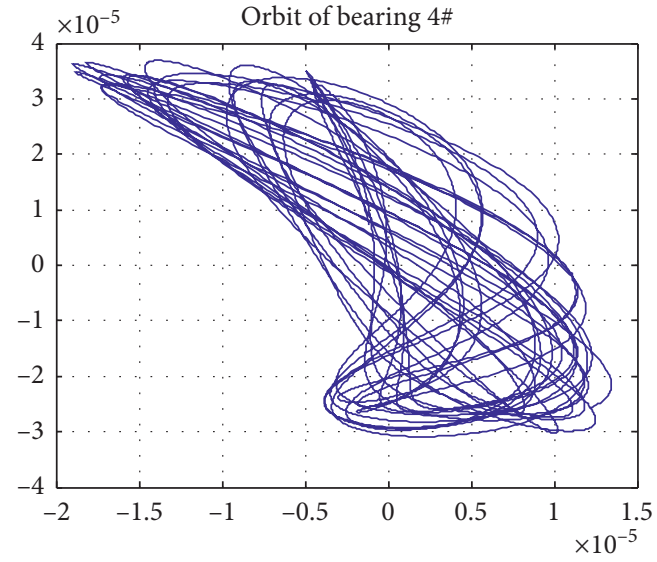

(b)

FIgURE 29: Orbits of bearings with misalignment $\left(\Delta=0.2 \mathrm{~mm}, \Omega_{1}=21.6 \mathrm{~Hz}, \Omega_{2}=34.6 \mathrm{~Hz}\right.$ ): (a) bearing 1 and (b) bearing 4 .

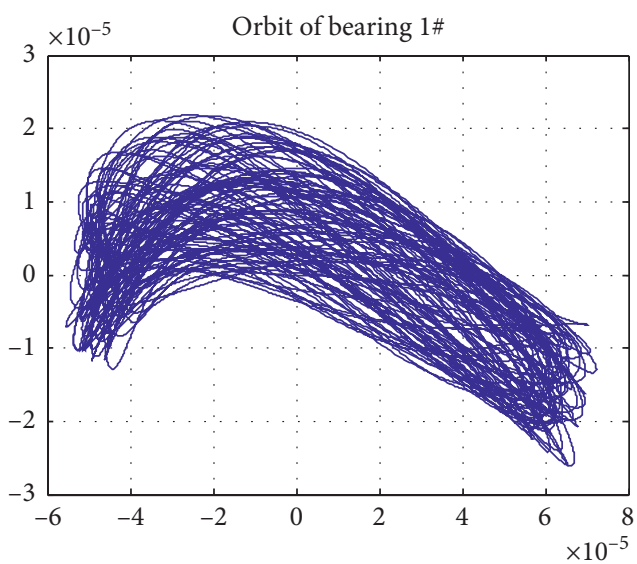

(a)

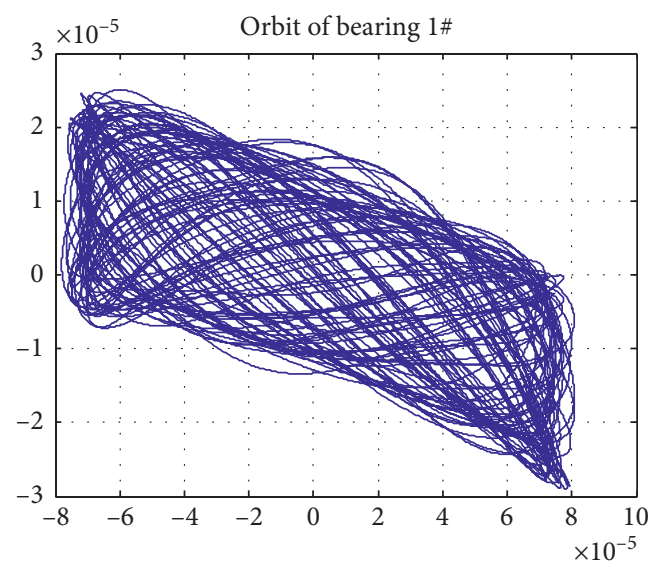

(b)

Figure 30: Orbits of bearing 1 at different misalignment $\left(\Omega_{1}=11.6 \mathrm{~Hz}, \Omega_{2}=18.6 \mathrm{~Hz}\right.$ ): (a) $\Delta=0.2 \mathrm{~mm}$ and (b) $\Delta=0.4 \mathrm{~mm}$.

\section{Conclusions}

In this paper, a finite element model for a dual-rotor system with a misaligned coupling is developed using the Timoshenko beam elements. The response of the system is generated using the Newmark- $\beta$ method. The spectral response of the system with both unbalances and misalignment has been studied. The experiments are performed to illustrate the theoretically developed characteristic vibration frequencies for a misaligned coupling in the dual-rotor system. Experimental and theoretical studies show a good agreement with each other, which verifies the analytical procedure adopted in this study. Some conclusions drawn from the study can be summarized as follows:

(1) The inner rotor with coupling misalignment makes both the inner and outer rotor appear $1 x, 2 x, 3 x$, and $4 x$ harmonic components, among which $1 \mathrm{x}$ and $2 \mathrm{x}$ are the main frequency components.

(2) Although the vibration caused by misalignment of the coupling in the inner rotor can be transmitted to the outer rotor, the closer the coupling is to the bearing, the more severe the vibration is. And the vibration amplitude of the misalignment direction is larger than that of the direction without misalignment.

(3) The amplitudes of each harmonic frequency increase with the increase of the misalignment.

(4) Harmonic resonance occurs when any harmonic frequencies of the misalignment response coincide with a natural frequency of the system. And the vibration will be intensified.

(5) The orbits of bearings tend to collapse toward a straight line for the angular misalignment. And the orbit diverges into a reticulated diamond shape from a straight line shape as the increase of misalignment.

\section{Data Availability}

The data supporting this research article are available from the corresponding author or first author on reasonable request. 


\section{Conflicts of Interest}

The authors declare that there are no conflicts of interest regarding the publication of this paper.

\section{Acknowledgments}

The authors would like to thank Dr. Qing Xiao for his valuable suggestions. The authors would also thank graduate students Zihao Liao and Liman Chen for their help in experiments. In addition, the authors would like to thank Linlin Song for her help in translation work. This work was supported by the Natural Science Foundation of China through the Grants 11672106, 11702091, and 51875196, the Natural Science Foundation of Hunan Province of China (2018JJ3140), and the Open Fund Project of Key Laboratory Hunan Province of Health Maintenance Mechanical Equipment (201702).

\section{References}

[1] L. Huang, H. Huang, and Y. Liu, "A fault diagnosis approach for rolling bearing based on wavelet packet decomposition and GMM-HMM," June 2019, vol. 24, no. 2, pp. 199-209, 2019.

[2] D. Yang, Y. Liu, S. Li, X. Li, and L. Ma, "Gear fault diagnosis based on support vector machine optimized by artificial bee colony algorithm," Mechanism and Machine Theory, vol. 90, pp. 219-229, 2015.

[3] D. Yang, Y. Liu, S. Li, J. Tao, C. Liu, and J. Yi, "Fatigue crack growth prediction of 7075 aluminum alloy based on the GMSVR model optimized by the artificial bee colony algorithm," Engineering Computations, vol. 34, no. 4, pp. 10341053, 2017.

[4] H. Ma, X. Tai, and Z. Zhang, "Dynamic characteristic analysis of a rotor system with rub-impact fault considering rotorstator misalignment," Przeglad Elektrotechniczny (Electrical Review), vol. 88, no. 9, pp. 145-149, 2012.

[5] A. W. Lees, J. K. Sinha, and M. I. Friswell, "Estimating rotor unbalance and misalignment from a single run-down," $M a$ terials Science Forum, vol. 440-441, pp. 229-236, 2003.

[6] M. Lal and R. Tiwari, "Experimental estimation of misalignment effects in rotor-bearing-coupling systems," in Proceedings of the 9th IFToMM International Conference on Rotor Dynamics, pp. 779-789, Springer, Basel, Switzerland, 2015.

[7] J. Rybczyski, "The possibility of evaluating turbo-set bearing misalignment defects on the basis of bearing trajectory features," Mechanical Systems and Signal Processing, vol. 25, no. 2, pp. 521-536, 2011.

[8] C. Verucchi, J. Bossio, G. Bossio, and G. Acosta, "Misalignment detection in induction motors with flexible coupling by means of estimated torque analysis and mcsa," Mechanical Systems and Signal Processing, vol. 80, pp. 570-581, 2016.

[9] M. C. Reddy and A. S. Sekhar, "Detection and monitoring of coupling misalignment in rotors using torque measurements," Measurement, vol. 61, pp. 111-122, 2015.

[10] X. X. Qi, M. L. Wang, L. J. Lin, J. J. Ji, and Q. K. Han, “Timefrequency characteristics analysis on vibration signals of a three-supported rotor system with misalignment," Advanced Engineering Forum, vol. 2, pp. 717-721, 2012.
[11] T. H. Patel and A. K. Darpe, "Vibration response of misaligned rotors," Journal of Sound and Vibration, vol. 325, no. 3, pp. 609-628, 2009.

[12] T. H. Patel and A. K. darpe, "Experimental investigations on vibration response of misaligned rotors," Mechanical Systems and Signal Processing, vol. 23, no. 7, pp. 2236-2252, 2009.

[13] T. H. Patel and A. K. Darpe, "Vibration response of a cracked rotor in presence of rotor-stator rub," Journal of Sound and Vibration, vol. 317, no. 3-5, pp. 841-865, 2008.

[14] M. A. Hili, T. Fakhfakh, L. Hammami, and M. Haddar, "Shaft misalignment effect on bearings dynamical behavior," The International Journal of Advanced Manufacturing Technology, vol. 26, no. 5-6, pp. 615-622, 2005.

[15] M. A. Hili, T. Fakhfakh, L. Hammami, and M. Haddar, "Angular misalignment effect on bearings dynamical behavior," The Arabian Journal for Science and Engineering, vol. 29, no. 1, pp. 69-80, 2004.

[16] M. Li, Z. Li, and X. Ping, "Nonlinear dynamic behaviors of rotor system with misaligned journal bearings," Applied Mechanics and Materials, vol. 271-272, no. 2181, pp. 10321038, 2013.

[17] Z. Li and M. Li, "Non-linear dynamics of a flexible multi-rotor bearing system with a fault of parallel misalignment," Applied Mechanics and Materials, vol. 138-139, no. 1520, pp. 104-110, 2012.

[18] A. Askarian and S. M. Hashemi, "Effect of axial force, unbalance and coupling misalignment on vibration of a rotor gas turbine," in Proceedings of the 14th International Congress on Sound \& Vibration, pp. 1-8, Cairns, Australia, July 2007.

[19] H. Li, Y. Chen, L. Hou, and Z. Zhang, "Periodic response analysis of a misaligned rotor system by harmonic balance method with alternating frequency/time domain technique," Science China Technological Sciences, vol. 59, no. 11, pp. 1717-1729, 2016.

[20] L. Ma, J.-H. Zhang, J.-W. lin, J. Wang, and X. Lu, "Dynamic characteristics analysis of a misaligned rotor-bearing system with squeeze film dampers," Journal of Zhejiang UniversityScience $a$, vol. 17, no. 8, pp. 614-631, 2016.

[21] K. M. Al-Hussain, "Dynamic stability of two rigid rotors connected by a flexible coupling with angular misalignment," Journal of Sound and Vibration, vol. 266, no. 2, pp. 217-234, 2003.

[22] J. Yi, B. Pang, H. Liu, F. Wang, B. Ji, and M. Jing, "Influence of misalignment on nonlinear dynamic characteristics for matched bearings-rotor system," Proceedings of the Institution of Mechanical Engineers, Part K: Journal of Multi-Body Dynamics, vol. 228, no. 2, pp. 172-181, 2014.

[23] J. H. Zhang, L. Ma, J. W. Lin, and G. C. Zhang, "Dynamic analysis of flexible rotor ball bearings system with unbalance misalignment rubbing coupling faults," Applied Mechanics and Materials, vol. 105-107, pp. 448-453, 2011.

[24] J. L. Nikolajsen, "The effect of misalignment on rotor vibrations," Journal of Engineering for Gas Turbines and Power, vol. 120, no. 3, pp. 635-640, 1998.

[25] C. B. Gibbons, "Coupling misalignment forces," in Proceedings of the 5th Turbomachinery Symposium, pp. 111-116, Texas A \& M University, College Station, TX, USA, 1976.

[26] A. S. Sekhar and B. S. Prabhu, "Effects of coupling misalignment on vibrations of rotating machinery," Journal of Sound and Vibration, vol. 185, no. 4, pp. 655-671, 1995.

[27] Y.-S. Lee and C.-W. Lee, "Modelling and vibration analysis of misaligned rotor-ball bearing systems," Journal of Sound and Vibration, vol. 224, no. 1, pp. 17-32, 1999. 
[28] S. Prabhakar, A. S. Sekhar, and A. R. Mohanty, "Crack versus coupling misalignment in a transient rotor system," Journal of Sound and Vibration, vol. 256, no. 4, pp. 773-786, 2002.

[29] A. K. Jalan and A. R. Mohanty, "Model based fault diagnosis of a rotor-bearing system for misalignment and unbalance under steady-state condition," Journal of Sound and Vibration, vol. 327, no. 3-5, pp. 604-622, 2009.

[30] W. Zhao, J. P. Jing, G. Meng, Y. Yang, and H.-Y. Bai, "Theoretical and experimental study on the dynamic response of multi-disk rotor system with flexible coupling misalignment," Proceedings of the Institution of Mechanical Engineers, Part C: Journal of Mechanical Engineering Science, vol. 226, no. 12, pp. 2874-2886, 2012.

[31] H. Ma, X. Wang, H. Niu, and B. Wen, "Oil-film instability simulation in an overhung rotor system with flexible coupling misalignment," Archive of Applied Mechanics, vol. 85, no. 7, pp. 893-907, 2015.

[32] D. L. Dewell and L. D. Mitchell, "Detection of a misaligned disk coupling using spectrum analysis," Journal of Vibration Acoustics Stress and Reliability in Design, vol. 106, no. 1, pp. 9-16, 1984.

[33] A. W. Lees, "Misalignment in rigidly coupled rotors," Journal of Sound and Vibration, vol. 305, no. 1-2, pp. 261-271, 2007.

[34] M. Xu and R. D. Marangoni, "Vibration analysis of a motorflexible coupling-rotor system subject to misalignment and unbalance, part I: theoretical model and analysis," Journal of Sound and Vibration, vol. 176, no. 5, pp. 663-679, 1994.

[35] M. Xu and R. D. Marangoni, "Vibration analysis of a motorflexible coupling-rotor system subject to misalignment and unbalance, part II: experimental validation," Journal of Sound and Vibration, vol. 176, no. 5, pp. 681-691, 1994.

[36] Z. Lu, X. Wang, L. Hou, Y. Chen, and H. Li, "Dynamic response analysis for the aero-engine dual-rotor-bearing system with flexible coupling misalignment faults," Journal of Vibroengineering, vol. 20, no. 5, pp. 2012-2026, 2018.

[37] J. Li, J. Hong, Y. Ma, and D. Zhang, "Modelling of misaligned rotor systems in aero-engines," in Proceedings of the ASME 2012 International Mechanical Engineering Congress \& Exposition, pp. 1-9, Houston, TX, USA, 2012.

[38] G. H. Wang, Y. H. Ma, T. Li, J. Li, and J. Hong, "Modelling of misaligned rotor system in aero-engines and interval method investigation," in Proceedings of the ASME 2013 International Mechanical Engineering Congress and Exposition, pp. 1-8, San Diego, CA, USA, 2013.

[39] C. Wang, Y. H. Ma, D. Y. Zhang, and J. Zhang, "Interval analysis on aero-engine rotor system with misalignment," in Proceedings of ASME Turbo Expo 2015: Turbine Technical Conference and Exposition, Montreal, Canada, August 2015.

[40] Z. Li, J. Jiang, and Z. Tian, "Non-linear vibration of an angular-misaligned rotor system with uncertain parameters," Journal of Vibration and Control, vol. 22, no. 1, pp. 129-144, 2016.

[41] D. X. Ren, J. Hong, and C. Wang, "Research on dynamic model of rotors with bearing misalignment," Applied Mechanics and Materials, vol. 539, no. 3066, pp. 3-8, 2014.

[42] N. Branch, Analysis of Spall Propagation in Case Hardened Hybrid Ball Bearings, pp. 20-21, University of Florida, Gainesville, FL, USA, 2010.

[43] M. I. friswell, J. E. T. Penny, and S. D. Garvey, Dynamics of Rotating Machines, pp. 141-310, Cambridge University Press, Cambridge, MA, USA, 2010.

[44] J. Piotrowski, Shaft Alignment Handbookpp. 353-366, CRC Press, Boca Raton, FL, USA, 3rd edition, 2007. 